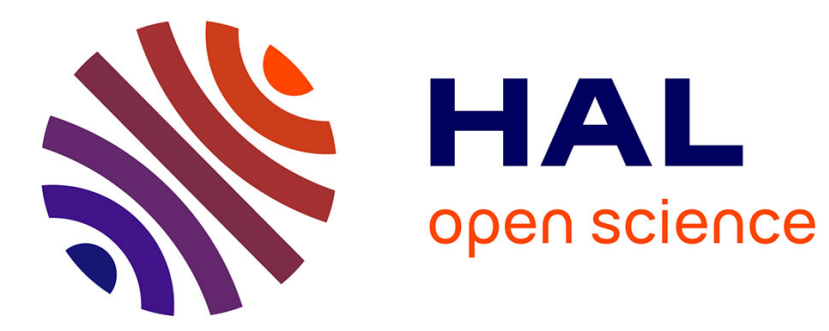

\title{
Polarizable Force Fields for Biomolecular Modeling
} Yue Shi, Pengyu Ren, Michael Schnieders, Jean-Philip Piquemal

\section{To cite this version:}

Yue Shi, Pengyu Ren, Michael Schnieders, Jean-Philip Piquemal. Polarizable Force Fields for Biomolecular Modeling. Reviews in Computational Chemistry, 28 (51), Wiley, 2015, 10.1002/9781118889886.ch2 . hal-01114184

\section{HAL Id: hal-01114184 https://hal.sorbonne-universite.fr/hal-01114184}

Submitted on 3 Nov 2015

HAL is a multi-disciplinary open access archive for the deposit and dissemination of scientific research documents, whether they are published or not. The documents may come from teaching and research institutions in France or abroad, or from public or private research centers.
L'archive ouverte pluridisciplinaire HAL, est destinée au dépôt et à la diffusion de documents scientifiques de niveau recherche, publiés ou non, émanant des établissements d'enseignement et de recherche français ou étrangers, des laboratoires publics ou privés. 


\section{Polarizable Force Fields for Biomolecular Modeling}

Yue Shi, Pengyu Ren ${ }^{\mathrm{a}}$

a. Department of Biomedical Engineering

The University of Texas at Austin

1 University Station, C0800

Austin, TX 78712

Michael Schnieders ${ }^{\mathrm{b}}$

b. Department of Biomedical Engineering, College of Engineering and Department of Biochemistry, Carver College of Medicine

The University of Iowa

Iowa City, Iowa, 52242

Jean-Philip Piquemal $^{\mathrm{c}}$

c. Laboratoire de Chimie Théorique (UMR 7616),

UPMC, Sorbonne Universités,

CC 137, 4 place Jussieu,

75252 Paris Cedex 05, France

23 


\section{1. Introduction}

25 Molecular mechanics based modeling has been widely used in the study of chemical and 26 biological systems. The classical potential energy functions and their parameters are 27 referred to as force fields. Empirical force fields for biomolecules emerged in the early 28 1970's, ${ }^{1}$ followed by the first molecular dynamics simulations of the bovine pancreatic 29 trypsin inhibitors (BPTI). ${ }^{2-4}$ Over the past 30 years, a great number of empirical 30 molecular mechanics force fields, including AMBER, ${ }^{5} \mathrm{CHARMM},{ }^{6}$ GROMOS, ${ }^{7}$ OPLS, ${ }^{8}$ 31 and many others, have been developed. These force fields share similar functional forms,

32 including valence interactions represented by harmonic oscillators, point dispersion33 repulsion for van der Waals (vdW) interactions, and an electrostatic contribution based 34 on fixed atomic partial charges. This generation of molecular mechanics force fields has 35 been widely used in the study of molecular structures, dynamics, interactions, design and 36 engineering. We refer interested readers to some recent reviews for detailed discussions. ${ }^{9}$ $37 \quad 10$

38 Although the fixed charge force fields enjoyed great success in many areas, there remains

39 much room for improvement. In fixed charge based electrostatic models, the atomic 40 partial charges are meant to be ñpre-polarizedò for condensed phases in an averaged

41 fashion, typically achieved by the fortuitous overestimation of electrostatic charges by

42 low-level ab initio quantum mechanics. Such models thus lack the ability to describe the 43 variation in electrostatics due to many-body polarization effects, which have been shown 44 to be a significant component of intermolecular forces. ${ }^{10-12}$ With the rapid growth of 45 computational resources, there has been increasing effort to explicitly incorporate many46 body induction into molecular mechanics to improve the accuracy of molecular modeling. 
47 Classical electrostatics models that take into account polarization appeared as early as the 48 1950s. Barker in his 1953 paper ñStatistical Mechanics of Interacting Dipolesò discussed 49 the electrostatic energy of molecules in terms of ñpermanent and induced dipolesò. ${ }^{13}$ 50 Currently, polarizable models generally fall into three categories: those based on induced 51 point dipoles, ${ }^{9,}{ }^{14-23}$ the classical Drude oscillators, ${ }^{24-26}$ and fluctuating charges. ${ }^{27-30}$ More 52 sophisticated force fields that are ñelectronic structure-basedò ${ }^{31,32}$ or use ñmachine 53 learning methodsơ ${ }^{33}$ also exist, but incur higher computational costs. Discussions of the 54 advantages and disadvantages of each model and their applications will be presented in 55 the following sections.

56 Compared to fixed charge models, the polarizable models are still in a relatively early 57 stage. Only in the past decade or so has there been a systematic effort to develop general 58 polarizable force fields for molecular modeling. A number of reviews have been 59 published to discuss various aspects of polarizable force fields and their development. ${ }^{9,34}$

$60{ }^{40}$ Here, we focus on the recent development and applications of different polarizable 61 force fields. We begin with a brief introduction to the basic principles and formulae 62 underlying alternative models. Next, the recent progress of several well-developed 63 polarizable force fields is reviewed. Finally, applications of polarizable models to a 64 range of molecular systems, including water and other small molecules, ion solvation, 65 peptides, proteins and lipid systems are presented.

\section{1. Modeling Polarization Effects}

67 1.1. Induced Dipole Models 
68 To describe electrostatic interactions involving polarization, we consider a system 69 consisting of a collection of charge distribution sites located at lone-pair positions, atomic 70 centers and/or molecular centers, depending on the resolution of the model. The total

71 charge distribution at site $i$ is the sum of permanent and induced charge

$$
\mathrm{M}_{\mathrm{i}}=\mathrm{M}_{\mathrm{i}}^{0}+\mathrm{M}_{\mathrm{i}}^{\mathrm{ind}}
$$

73 where $\boldsymbol{M}$ represents the charge distribution. This distribution can be a simple point charge,

74 a point multipole expansion with charge, dipole, quadrupole and/or higher order moments,

75 or a continuous charge distribution. While the principles described below are not limited

76 to any particular representation of charge distribution, we will use point multipoles for

77 convenience.

78 The electrostatic interaction energy between two charge sites $i$ and $j$ is given by

$$
\mathrm{U}_{\oplus \mathrm{le}}=\frac{1}{2} \sum_{\mathrm{i}} \sum_{\mathrm{j} \neq 1} \mathrm{M}_{1}^{\mathrm{t}} \mathrm{T}_{\mathbf{1 j}} \mathrm{M}_{\mathrm{j}}
$$

80

81

82

83

84

$$
\mathrm{U}_{\text {work }}=\frac{1}{2} \sum_{\mathrm{i}}\left(\mathrm{M}_{1}^{\text {ind }}\right)^{\mathrm{t}} \alpha_{1}^{-1} \mathrm{M}_{1}^{\mathrm{ind}}
$$

85 where $\breve{\mathbf{U}}$ is the polarizability of site $i$ that includes all orders of polarizability including 86 dipole polarizability. ${ }^{41}$ Although $\breve{\mathbf{U}}$ is generally treated as an isotropic quantity, as in the 
87 Applequist scheme ${ }^{41}$, ab initio anisotropic polarizability tensors can be derived from 88 quantum mechanical calculations. ${ }^{42,43}$

89 The total electrostatic energy is

$$
\mathrm{U}_{\mathrm{ele}}=\frac{1}{2} \Sigma_{\mathrm{l}} \Sigma_{\mathrm{j} \neq 1} \mathrm{M}_{1}^{\mathrm{t}} \mathrm{T}_{\mathrm{l}]} \mathrm{M}_{\mathrm{j}}+\frac{1}{2} \Sigma_{\mathrm{l}}\left(\mathrm{M}_{1}^{\mathrm{ind}}\right)^{\mathrm{t}} \alpha_{1}^{-1} \mathrm{M}_{1}^{\mathrm{lnd}}
$$

91

92

93

97

98 where the first term is the permanent electrostatic energy and the second term is the 101 polarization energy.

\subsection{Classic Drude Oscillators}

In the Drude oscillator model, the polarization effect is described by a point charge (the

104 Drude oscillator) attached to each non-hydrogen atom via a harmonic spring. The point 

charge can move relative to the attachment site in response to the electrostatic environment. The electrostatic energy is the sum of the pairwise interactions between

107 atomic charges and the partial charge of the Drude particles

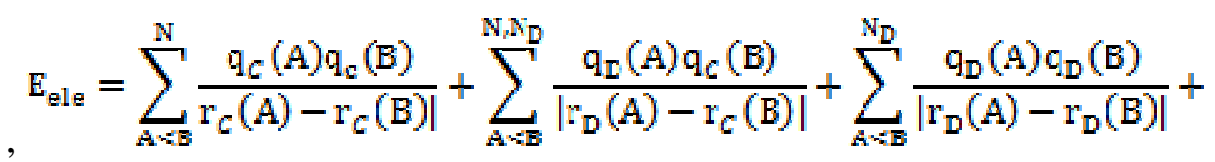

$$
\frac{1}{2} \sum_{\mathrm{A}}^{\mathrm{N}_{\mathrm{D}}} \mathrm{k}_{\mathrm{D}}\left(\mathrm{r}_{\mathrm{D}}(\mathrm{A})-\mathrm{r}_{\mathrm{C}}(\mathrm{B})\right)^{2}
$$

110

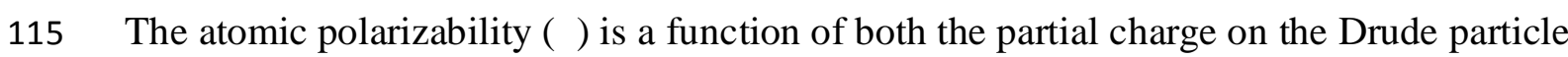
116 and the force constant of the spring

$$
\alpha=\frac{q_{D}^{2}(A)}{k_{D}}
$$

118 Both the induced-dipole and Drude oscillator approaches benefit from short-range Thole 119 damping to avoid a polarization catastrophe and to produce an anisotropic molecular 120 polarization response. ${ }^{45}$

\section{$121 \quad$ 1.3. Fluctuating Charges}


122 The formalism of the fluctuating charge model is based on the charge equilibration

123 (CHEQ) method, ${ }^{46}$ in which the chemical potential is equilibrated via the redistribution of

124 charge density. The charge-dependent energy for a system of $M$ molecules containing $N_{i}$

125 atoms per molecule is expressed as

126

128 where $Q_{i}$ is the partial charge on atomic site $i$. The $\mathrm{G}$ describes the atomic

129 electronegativity controlling the directionality of electron flow, and $J$ is the atomic

130 hardness that represents the resistance to electron flow to or from the atom. These

131 parameters are optimized to reproduce molecular dipoles and the molecular polarization

132 response. The charge degrees of freedom are typically propagated via an extended

133 Lagrangian formulation: ${ }^{47}$

134

$L=\sum_{i=1}^{\mathrm{M}} \sum_{\alpha=1}^{\mathrm{N}} \frac{1}{2} \mathrm{~m}_{\mathrm{i \alpha}}\left(\frac{\mathrm{dr} \mathrm{r}_{\mathrm{i \alpha}}}{\mathrm{dt}}\right)^{2}+\sum_{\mathrm{l}=1}^{\mathrm{M}} \sum_{\alpha=1}^{\mathrm{N}} \frac{1}{2} \mathrm{~m}_{\mathrm{Q}, \mathrm{i \alpha}}\left(\frac{\mathrm{dQ} \mathrm{Q}_{1 \alpha}}{\mathrm{dt}}\right)^{2}-\mathrm{E}(\mathrm{Q}, \mathbf{r})-\sum_{\mathrm{l}=1}^{\mathrm{M}} \lambda_{\mathrm{i}} \sum_{\alpha=1}^{\mathrm{N}} \mathrm{Q}_{\mathrm{i \alpha}}[11]$

135 where the first two terms represent the nuclear and charge kinetic energies, the third term

136 is the potential energy, and the fourth term is the molecular charge neutrality constraint

137 enforced on each molecule $i$ via a Lagrange multiplier $x_{i}$ The extended Lagrangian 138 approach can also be applied to the induced dipole and Drude oscillator models described 139 earlier. While the extended Lagrangian seems to be more efficient than the iterative 140 method, fictitious masses and smaller time-steps are required to minimize the coupling 
between the polarization and atomic degrees of freedom, which can never be completely

142 eliminated. $^{44}$

143 A few general force fields have been developed based on these formulas to explicitly

144 treat the polarization effect. We now discuss development highlights for some of the 145 representative force fields.

\section{2. Recent Developments}

\section{2.1. AMOEBA}

148 The AMOEBA (Atomic Multipole Optimized Energetics for Biomolecular Applications) 149 force field, developed by Ponder, Ren and co-workers, ${ }^{15,18,37}$ utilizes atomic multipoles 150 to represent permanent electrostatics and induced atomic dipoles for many-body 151 polarization. The valence interactions include bond, angle, torsion and out-of-plane 152 contributions using typical molecular mechanics functional forms. The van der Waals 153 interaction is described by a buffered-14-7 function. The atomic multipole moments 154 consist of charge, dipole and quadrupole moments, which are derived from ab initio 155 quantum mechanical calculations using procedures such as Stoneô Distributed Multipole 156 Analysis (DMA). ${ }^{48-50}$ The higher order moments make possible anisotropic 157 representations of the electrostatic potential outside atoms and molecules. The 158 polarization effect is explicitly taken into account via atomic dipole induction. The 159 combination of permanent atomic multipoles and induced dipoles enables AMOEBA to 160 capture electrostatic interactions in both gas and condensed phase accurately. The vdW 161 parameters of AMOEBA are optimized simultaneously against both ab initio gas-phase 162 data and condensed-phase experimental properties. 
163 In the past decade, AMOEB A has been applied to the study of water, ${ }^{15}$ monovalent and 164 divalent ions, ${ }^{51-53}$ small molecules, ${ }^{54,55}$ peptides $^{18,} 56$ and proteins. ${ }^{57-59}$ AMOEBA 165 demonstrated that a polarizable force field is able to perform well in both gas and 166 solution phases with a single set of parameters. In addition, AMOEBA is the first 167 general-purpose polarizable force field utilized in molecular dynamics simulations of 168 protein-ligand binding and calculation of absolute and relative binding free energies. ${ }^{58-62}$ 169 The computed binding free energies between trypsin and benzamidine derivatives 170 suggests significant non-additive electrostatic interactions as the ligand desolvates from 171 water and enters the protein pocket (see Section 4.4 for further discussion). AMOEBA 172 has recently been extended to biomolecular X-ray crystallography refinement ${ }^{63,64}$, and 173 consistently successful prediction of the structure, thermodynamic stability and solubility 174 of organic crystals ${ }^{65}$ are encouraging.

175 AMOEBA has been implemented in several widely used software packages including 176 TINKER, ${ }^{66}$ OpenMM,${ }^{67}$ Amber, ${ }^{68}$ and Force Field $\mathrm{X} .{ }^{69}$ The AMOEBA polarizable force 177 field was first implemented within the FORTRAN-based TINKER software package ${ }^{70}$ 178 using Particle Mesh Ewald (PME) for long-range electrostatics. Implementation of the 179 polarizable-multipole Poisson-Boltzmann, ${ }^{71}$ which depends on the Adaptive Poisson180 Boltzmann Solver (APBS), ${ }^{72}$ and generalized Kirkwood $^{73}$ continuum electrostatics 181 models also exist in TINKER, which is now being parallelized using OpenMP. The 182 algorithms in TINKER are also available from within CHARMM using the MSCALE 183 interface. ${ }^{74,75}$ Alternative FORTRAN implementations of AMOEBA using PME are 184 available in the Sander and PMEMD molecular dynamics engines of AMBER, ${ }^{68}$ with the 185 latter parallelized using MPI. The PME treatment of AMOEBA electrostatics has recently 

been extended within the Java Runtime Environment (JRE) program Force Field $X$ by

187 incorporating explicit support for crystal space group symmetry, ${ }^{63}$ parallelizing for 188 heterogeneous computer hardware environments ${ }^{63}$ and supporting advanced free energy 189 methods such as the Orthogonal Space Random Walk (OSRW) strategy. ${ }^{65,} 76$ These 190 advancements are critical for applications such as AMOEBA-assisted biomolecular X-ray 191 refinement, ${ }^{63,} 77$ efficient computation of protein-ligand binding affinity, ${ }^{57,61}$ and 192 prediction of the structure, stability and solubility of organic crystals. ${ }^{65}$ Finally, the 193 OpenMM software is working toward a general implementation of AMOEBA using the 194 CUDA GPU programming language. ${ }^{78}$

\section{2.2. SIBFA}

196 The SIBFA (Sum of Interactions Between Fragments Ab initio computed) force field for

197 small molecules and flexible proteins, developed by Gresh, Piquemal et. $a l^{79-83}$ is one of 198 the most sophisticated polarizable force fields because it incorporates polarization, 199 electrostatic penetration ${ }^{84}$ and charge-transfer effects. ${ }^{85}$

200 The polarization is treated with an induced dipole model, in which the distributed 201 anisotropic polarizability tensors ${ }^{43}$ are placed on the bond centers and on the heteroatom 202 lone pairs. Quadrupolar polarizabilities are used to treat metal centers. The force field is 203 designed to enable the simultaneous and reliable computation of both intermolecular and 204 conformational energies governing the binding specificities of biologically and 205 pharmacologically relevant molecules. Similar to AMOEBA, permanent multipoles are 206 used for permanent electrostatics in SIBFA. Flexible molecules are modeled by 207 combining the constitutive rigid fragments. SIBFA is formulated on the basis of quantum 
chemistry and calibrated on energy decomposition analysis, as oppose to AMOEBA

209 which relies more on condensed-phase experimental data. It aims to produce accurate

210 interaction energy comparable with $a b$ initio results. The development of SIBFA

211 emphasizes separability, anisotropy, nonadditivity and transferability. The analytical

212 gradients for charge-transfer energy and solvation contribution are not yet available in

213 SIBFA although molecular dynamics simulations with a simplified potential have been

214 attempted and will be reported in the near future.

215 SIBFA has been validated on a wide range of molecular systems from water clusters ${ }^{86}$ to

216 large complexes like metalloenzymes encompassing $\mathrm{Zn}$ (II). ${ }^{87-92}$ It has been used to

217 investigate molecular recognition problems including the binding of nucleic acids to

218 metal ions, ${ }^{93-95}$ the prediction of oligopeptide conformations, ${ }^{86,}, 96$ and for ligand-protein

219 binding. ${ }^{97}$ Most of the SIBFA calculations reproduced closely the quantum chemistry

220 results, including both the interaction energy and the decomposed energy terms. At the

221 same time, electrostatic parameters are demonstrated to be transferable between similar

222 molecules.

223 ,A Gaussian based electrostatic model (GEM) has been explored as an alternative to

224 distributed point multipole electrostatic representation. ${ }^{98}$ GEM computes the molecular

225 interaction energies using an approach similar to SIBFA but replacing distributed

226 multipoles by electron densities. ${ }^{99}$ GEM better captures the short-range effects on

227 intermolecular interaction energies, and it naturally includes the penetration effect.

228 Calculations on a few simple systems like water clusters ${ }^{99}$ have demonstrated GEMôs

229 capability to reproduce quantum chemistry results. Furthermore, implementating PME

230 for GEM in a PBC showed reasonable computational efficiency thanks to the use of 
231 Hermite Gaussian functions. ${ }^{100}$ Therefore, replacing SIBFAô distributed multipoles with

232 the GEM continuous electrostatic model will be a future direction of methodology

233 development. ${ }^{98}$

\section{$234 \quad$ 2.3. NEMO}

235 NEMO (Non-Empirical Molecular Orbital) is a polarizable potential developed by 236 Karlström and co-workers. ${ }^{101-103}$ The NEMO potential energy function is composed of 237 electrostatics, induction, dispersion and repulsion terms. The induction component is 238 modeled using induced pointï dipole moments with recent addition of induced pointï 239 quadrupole moments. ${ }^{22}$ The electrostatics, previously represented by atomic charges and 240 dipoles, has also been extended to include atomic quadrupole moments leading to notable 241 improvement on formaldehyde. The atomic multipole moments are now obtained from ab 242 initio calculation using a LoProp procedure. ${ }^{104}$ The LoProp is claimed to provide atomic 243 multipoles and atomic polarizabilities that are less sensitive to basis sets than are other 244 methods such as Distributed Multipole Analysis (DMA). Also, NEMO is the only force 245 field that explores the possibility of including interactions between permanent multipoles 246 and higher-order induced multipoles involving higher-order hyperpolarizabilities. ${ }^{22}$

247 NEMO has demonstrated its ability to describe accurately both inter and intramolecular 248 interactions in small systems, including: glycine dipeptide conformation profiles, ${ }^{105}$ ion249 water droplets, ${ }^{106}$ and urea transition from nonplanar to planar conformation in water. ${ }^{107}$ 250 Its applicability to biomacromolecules is not yet known.

\section{2.4. CHARMM-Drude}


252 In addition to the induced dipole model, the classical Drude oscillator model is another 253 popular approach for modeling polarization effects. ${ }^{39,}{ }^{108}$ Roux, MacKerell and their 254 colleagues have been developing a polarizable CHARMM force field based on this 255 approach. ${ }^{25,26,109,117}$ Unlike the induced dipole model, which treats the polarization 256 response using point dipoles, the Drude model represents the polarizable centers by a pair 257 of point charges. A point partial charge is tethered via a harmonic spring for each non258 hydrogen atom. This point charge (the Drude oscillator) can react to the electrostatic 259 environment and cause the displacement of the local electron density. The atomic 260 polarizability depends on both the Drude particle charge and the harmonic force constant. 261 In MD simulations, the extended Lagrangian is used to evaluate the polarization response, 262 by allowing the Drude particles to move dynamically and experience nonzero forces. 263 Small fictitious masses are assigned to each Drude particle and independent low 264 temperature thermostats are applied to the Drude particle degrees of freedom. ${ }^{118}$ In case 265 of energy minimization, self-consistent iteration will be required to solve for the 266 polarization.

267 Determining electrostatic parameters for the Drude oscillator is not as straightforward as 268 for induced dipole models. Masses assigned to the Drude particles are chosen empirically. 269 The values for atomic charges and polarizabilities requires a series of calculations of 270 perturbed ESP maps. This force field has been parameterized for water ${ }^{25,26}$, and for a 271 series of organic molecules including: alkanes, ${ }^{110}$ alcohols, ${ }^{111}$ aromatics, ${ }^{112}$ ethers, ${ }^{113,} 114$ 272 amides, ${ }^{109}$ sulfurs, ${ }^{115}$ and ions. ${ }^{119,}{ }^{120}$ An attempt has also been made to combine the 273 Drude-based polarizable force field with quantum mechanics in QM/MM methods. ${ }^{121}$ It 274 was noted that pair-specific vdW parameters are needed to obtain accurate hydration free 
275 energies of small molecules using the polarizable force field. This is likely due to the

276 problematic combining rules used to compute the vdW interactions between unlike atoms.

277 The Drude model has been implemented in $\mathrm{CHARMM}^{74,122}$ and in the NAMD

278 package, ${ }^{123}$ in which the computational cost is about 1.2 to 1.8 times greater than that of

279 fixed-charge CHARMM. ${ }^{124}$

\section{2.5. CHARMM-FQ}

281 The fluctuating charge model (FQ), also known as charge equilibration or 282 electronegativity equalization model, is an empirical approach for calculating charge 283 distributions in molecules. In this formalism, the partial charge on each atom is allowed 284 to change to adapt to different electrostatic environments. The variable partial charges are 285 computed by minimizing the electrostatic energy for a given molecular geometry. 286 Compared with the induced dipole and Drude models, the fluctuating charge models are 287 minimally parameterized and easier to implement because the polarizability is induced 288 without introducing new interactions beyond the point charges. Either extended 289 Lagrangian or self-consistent iteration can be used to compute the fluctuating charges in 290 MD simulations, with similar advantages and disadvantages as discussed above.

291 The CHARMM-FQ force field, ${ }^{125,126}$ developed by Patel, Brooks, and their coworkers, 292 has been parameterized for small molecules, ${ }^{28}$ proteins, ${ }^{28,}{ }^{127}$ lipids, lipid bilayers, ${ }^{113,} 128$ 293 and carbohydrates. ${ }^{125}$ The force field has been applied to investigate liquidï vapor 294 interfaces in addition to biophysical studies. ${ }^{129}$ There are some known limitations for 295 fluctuating charge models, however, such models allow artificial charge transfer between 296 widely separated atoms but that can be controlled with additional constraints. Also the 
297 intramolecular charge-flow is limited by the chemical connectivity. It is thus difficult to 298 capture the out-of-plane polarization in molecules such as aromatic benzenes with 299 additional charge sites. The CHARMM-FQ force field has been implemented in the 300 CHARMM software package. ${ }^{74}$

\section{$301 \quad$ 2.6. X-Pol}

302 Gao and coworkers proposed the X-Pol framework by combining the fragment-based 303 electronic structure theory with a molecular mechanical force field. ${ }^{31,32,130}$ Unlike the 304 traditional force fields, X-Pol does not require bond stretching, angle, and torsion terms 305 because they are represented explicitly by quantum mechanics. The polarization and 306 charge transfer between fragments are also evaluated quantum mechanically. ${ }^{130}$ 307 Furthermore, X-Pol can be used to model chemical reactions.

308 In X-Pol, large molecular systems are divided into small fragments. Electrostatic 309 interactions within the fragments are treated using the electronic structure theory. The 310 electrostatic interactions between fragments are described by the combined quantum 311 mechanical and molecular mechanical (QM/MM) approach. Also, a vdW term is added to 312 the interfragment interaction as a consequence of omitting electron correlation and 313 exchange repulsion. A double self-consistent-field (DSCF) procedure is used to converge 314 the total electronic energy of the system as well as the energy within the fragments (this 315 includes the mutual polarization effect).

316 The X-Pol potential has been applied to MD simulations of liquid water, ${ }^{131}$ liquid 317 hydrogen fluoride, ${ }^{132}$ and covalently bonded fragments. ${ }^{133,134}$ This model was recently 318 used in a molecular dynamics simulation of a solvated protein. ${ }^{135}$ As expected the 
computational efficiency of the X-Pol is in between that of a simple classical force field

320 and a full $a b$ initio method. The solvated trypsin required $62.6 \mathrm{~h}$ to run a $5 \mathrm{ps}$ simulation

321 on a single $1.5 \mathrm{GHz}$ IBM Power4 processor. A parallel version of X-Pol is being

322 developed.

\section{2.7. PFF}

324 Kaminski et al. developed a polarizable protein force field (PFF) based on ab initio 325 quantum theory. ${ }^{136,137}$ The electrostatic interaction is modeled with induced dipoles and 326 permanent point charges. With the exception of a dispersion parameter, all other 327 parameters, including the electrostatic charges and polarizabilities, are obtained by fitting 328 to quantum chemical binding energy calculations for homodimers. The dispersion 329 parameters are later refined by fitting to the experimental densities of organic liquids. ${ }^{16}$ 330 Gas-phase many-body effects, as well as conformational energies, are well reproduced, ${ }^{137}$ 331 and MD simulations for real proteins are reasonably accurate at modest computational 332 costs. $^{16,138}$

333 To reduce the computational cost, a POSSIM (Polarizable Simulations with Second-order 334 Interaction Model) force field was later proposed, in which the calculation of induced 335 dipoles stops after one iteration. ${ }^{139,} 140$ The computational efficiency can be improved by 336 almost an order of magnitude by using this formalism. Because the analytical gradients 337 (forces) are unavailable, a Monte-Carlo technique is used in condensed-phase simulations. 338 POSSIM has been validated on selected small model systems, showing good agreement 339 with ab initio quantum mechanical and experimental data. Parameters for alanine and 340 protein backbone have been reported. ${ }^{141}$ 
341 Polarizable force fields for non-biological systems also exist. A many-body polarizable 342 force field by Smith and coworkers was developed and applied to the simulations of ion 343 conduction in polyethylene oxide (PEO). ${ }^{142-144}$ Cummings and coworkers developed an 344 interesting Gaussian charge polarizable force field for ions and in polyethylene oxide

345 (PEO). ${ }^{145-147}$ A polarizable force field for ionic liquids was also reported to provide 346 accurate thermodynamics and transport properties. ${ }^{148}$

\section{3. Applications}

\section{$348 \quad 3.1 . \quad$ Water Simulations}

349 Due to its important role in life, water is a natural choice for polarizable force field 350 development. After the polarizable (and dissociable) water model of Stillinger and 351 David, ${ }^{149}$ more than a dozen polarizable water models have been reported. ${ }^{150}$

352 Similar to how the polarization models discussed previously, the polarizable water 353 models likewise fall into three major categories. Most belong to the first category, 354 including the Stillinger and Davidê water model, SPCP, ${ }^{151}$ PTIP4P, ${ }^{152} \mathrm{CKL},{ }^{153} \mathrm{NCC},{ }^{154}$ 355 PROL, ${ }^{155}$ Dang-Chang ${ }^{156}$ and others. These models all adopted the induced dipole 356 framework to treat polarization, typically using a single polarizable site on water. TTM 357 models ${ }^{157-160}$ and the AMOEBA water mode ${ }^{15}$ utilize an interactive, distributed atomic 358 polarizability with Tholeôs damping scheme ${ }^{45}$ to treat electrostatics and polarization. The 359 Drude Oscillator-based water models include SWM4-DP, ${ }^{26}$ and SWM4-NDP, ${ }^{25}$ as well as 360 the Charge-On-Spring (COS) model, ${ }^{161}$ and its improved variation. ${ }^{162}$ The third group 361 includes the SPC-FQ and TIP4P-FQ ${ }^{163}$ water models that utilize the fluctuating charge 362 scheme to model polarization. The partial charges flow from one atom to another, and the 
total charge of a water molecule need not be zero. Stern et al. proposed a unique water

364 model (POL5) by combining the fluctuating charge with the point induced dipole

365 scheme. ${ }^{164}$ Several more sophisticated polarizable water models based on quantum

366 mechanics were developed based on quantum mechanics, including QMPFF, ${ }^{165}$ DPP2, ${ }^{166}$

367 and Polarflex. ${ }^{167}$ For example, the charge penetration, induction, and charge transfer

368 effects have been incorporated into the DPP2 (Distributed Point Polarizable Model)

369 model which reproduces well the high-level $a b$ initio energetics and structures for large

370 water clusters.

371 An advantage of a polarizable water model over most non-polarizable models is the

372 ability to describe the structure and energetics of water in both gas and condensed phases.

373 Water dimer interaction energies, the geometry of water clusters and the heat of

374 vaporization of neat water can be reproduced well by most polarizable models. Some

375 highly parameterized nonpolarizable force fields such as TIP5P, TIP4P-EW and

376 TIP4P/2005 actually perform as well or better than some polarizable force fields over a

377 range of liquid properties, including the density-temperature profile, radial distribution

378 function, and diffusion coefficient. However, for water molecules experiencing

379 significant changes in environment, e.g., from bulk water to the vicinity of ions or 380 nonpolar molecules, only the polarizable models can capture the change of water dipole,

381 structure and energetics. ${ }^{168}$

382 Polarization water models are being extended and applied to other phases as well as to

383 the interface between different phases. Rick et al recently incorporated charge transfer

384 into their polarizable water model that was then used to study ice/water coexistence

385 properties and properties of the ice Ih phase. ${ }^{169}$ The POL3 water model ${ }^{14,170}$ was used to 
study the ice-vapor interface, and to calculate the melting point of ice Ih. Bauer and Patel

387 used the TIP4P-QP model to study the liquid-vapor coexistence. ${ }^{171}$

\section{$388 \quad 3.2 . \quad$ Ion Solvation}

389 Ions are an important component in many chemical and biological systems. Nearly half 390 of all proteins contain metal ions, and they play essential roles in many fundamental 391 biological functions. Some metal ions are critical for both protein structure and function.

392 In enzymes, ions can bind and orient the substrates through electrostatic interactions at 393 the active sites, thus controlling catalytic reaction. Divalent ions are vital in nucleic acid 394 structures. Modeling ion-water and ion-biomolecule interactions accurately is very 395 important.

396 Due to the high electron density and small sizes of ions, the non-polarizable models fail 397 to capture the structural details adequately and do not or to reproduce the atomic dipole 398 of water around the ions. ${ }^{172-176}$ Several studies of ion solvation have been reported using 399 different polarizable models ${ }^{51-53}, 116,120,177-187$ with analyses focused on solvation 400 structures, charge distribution, and binding energies. Noted that no straightforward 401 experimental measurement of hydration free energy data exist because the macroscopic 402 system must be neutral. Different assumptions are used to decompose the experimental 403 hydration free energy into single ion contributions. The hydration free energy of some 404 monovalent ions such as $\mathrm{Na}^{+}$and $\mathrm{K}^{+}$from different sources can vary by as much as 10 $405 \mathrm{kcal} / \mathrm{mol}$. It is more reliable to compare the hydration free energy of the whole salt and 406 the relative energy between cations or anions. 
407 The AMOEBA polarizable force field has been used to model a number of anions and 408 cations, including $\mathrm{Na}^{+}, \mathrm{K}^{+}, \mathrm{Mg}^{++}, \mathrm{Ca}^{++}, \mathrm{Zn}^{++}, \mathrm{Cl}^{-}, \mathrm{Br}^{-}$, and $\mathrm{I}^{-} \cdot{ }^{51-53,188}$ Parameters for these 409 ions, including the vdW parameters and polarization damping coefficients (for divalent 410 ions only), were obtained by fitting to the ab initio QM interaction energy profiles of ion-

411 water pairs. Molecular dynamics simulations were then performed to evaluate the ion412 cluster solvation enthalpies and solvation free energies. ${ }^{51-53,188}$ The excellent agreement 413 between calculated and experimental hydration free energy, often within $1 \%$, demonstrate 414 that polarizable force fields are transferable between phases. A $b$ initio energy 415 decomposition using, e.g., the Constrained Space Orbital Variations (CSOV) method, ${ }^{99}$, $416{ }^{189}$ have also been applied to examine the polarization component of the ion-water 417 interaction energy and to guide the force field parameterization. ${ }^{53,190}$ More recently, the 418 AMOEBA force field was used to model the hydration of high valent $\operatorname{Th}(\mathrm{IV})^{94}$ and 419 studies on open-shell actinides are underway.

420 The SIBFA model was used to examine $\mathrm{Pb}(\mathrm{II}),{ }^{191}$ lanthanides ( $\mathrm{La}(\mathrm{III})$ and $\mathrm{Lu}(\mathrm{III})$ ) and 421 actinides (Th(IV)) in water. ${ }^{94}$ SIBFA-predicted interaction energies generally matched 422 well with the $a b$ initio results, including the energy decompositions. Lamoureux and 423 Roux developed the CHARMM polarizable force field for alkali and halide ions based on 424 the Drude Oscillator. ${ }^{177}$ Hydration free energies, calculated via thermodynamic 425 integration, ${ }^{192}$ showed an encouraging agreement with experiment.

\section{$426 \quad 3.3 . \quad$ Small Molecules}

427 Small molecules are building blocks of biomolecules and serve as substrates and 428 inhibitors. Abundant experimental measurements on various physical and chemical 
429 properties exist for common organic molecules which in turn are used in the 430 parameterization of the force fields. Polarizable and non-polarizable force fields can 431 usually produce reasonable estimations of physical properties of neat liquids. ${ }^{193-196}$ 432 Extensive studies using polarizable force fields, covering major functional group, 433 including alkanes, alcohols, aldehydes, ketones, ethers, acids, aromatic compounds, 434 amines, amides, and some halogen compounds have been reported. ${ }^{28,36,55,110,112,126,197-}$ $435{ }^{199}$ Calculations of structure, dipole moment, heterodimer binding energy, liquid diffusion 436 constant, density, heat of vaporization, and hydration free energy are usually performed 437 to assess the quality of force field parameters.

438 The electrostatic multipole parameters in AMOEBA were derived using the DMA 439 procedure. They can be further optimized to the electrostatic potentials of chosen $a b$ 440 initio theory and basis sets. The AMOEBA valence parameters were derived from $a b$ 441 initio data such as molecular geometries and vibrational frequencies of the gas-phase 442 monomer. The vdW parameters are estimated from gas-phase cluster calculations, and 443 subsequently refined in liquid simulations using experimental data (e.g., densities and 444 heats of vaporization). The torsional parameters the last obtained during the 445 parameterization scheme are derived by fitting to ab initio QM conformational energy 446 profiles. An automated protocol (PolType) that can generate AMOEBA parameters for 447 small molecules is under development. ${ }^{200}$ Because force field parameterization is a 448 tedious process, such an automated tool is convenient and reduces the likelihood of 449 human error.

450 The CHARMM-Drude force field developers devoted much of their efforts on organic 451 compounds. Their parameterization scheme starts from an initial guess of charge (based 
452 on the CHARMM22 force field), and invokes changes at some lone pair sites. Those 453 parameters are then fit to a series of ñperturbedòESP maps. The vdW parameters are then 454 optimized to match neat liquid properties as is done many other force fields. ${ }^{115}$ Overall, a 455 systematic improvement over the CHARMM22 additive force field has been observed for 456 both gas-phase and condensed-phase properties. These studies on small molecules lay the 457 groundwork for developing a Drude-based polarizable force field for proteins and nucleic 458 acids

\section{3.4. Proteins}

460 One of the goals for polarizable force fields is to model accurately protein structures, 461 dynamics, and interactions. Proteins are a ubiquitous class of biopolymers whose 462 functionalities depend on the details of their 3D structures, which, in turn, are largely 463 determined by their amino acid sequences. Fixed-charge force fields for proteins, like 464 AMBER, CHARMM, and OPLS-AA, have been developed and for years subjected to 465 various tests and validations. The development of polarizable protein force fields is still 466 in its infancy. Although the importance of including polarization effects was recognized 467 long ago, polarizable protein force fields emerged only in the past decade. $9,21,28,29,37,138$, 201-205

469 The use of polarizable electrostatics in protein simulations dates back to $1976,{ }^{1}$ when 470 Warshel and Levitt simulated lysozyme via single point calculations. Kaminski et al. 471 reported in 2002 an ab initio polarizable protein force field (PFF) based on inducible 472 dipoles and point charges. ${ }^{16,137}$ Simulations on bovine pancreatic trypsin inhibitor using 473 PDFF showed a satisfactory root mean square displacement (RMSD) compared to the 
474 experimental crystal structure and polarization was found to affect the solvation 475 dynamics. ${ }^{138}$ The fluctuating-charge based ABEEM/MM force field was used to examine 476 protein systems like trypsin inhibitors ${ }^{206}$ and the heme prosthetic group. ${ }^{207}$ The SIBFA

477 force field has been used to study the interaction between focal adhesion kinase (FAK) 478 and five pyrrolopyrimidine inhibitors. ${ }^{208}$ The energy balances accounting for the 479 solvation/desolvation effects calculated by SIBFA agree with experimental ordering. 480 Water networks in the binding pocket were shown to be critical in terms of binding 481 affinity. Moreover, the polarization contribution was considered as an indispensable 482 component during the molecular recognition. In comparison, the continuum reaction field 483 procedure fails to reproduce these properties. In addition kinases, the SIBFA protein 484 force field has been used to study a variety of metalloproteins encompassing cations such 485 as $\mathrm{Cu}^{+}, \mathrm{Zn}^{++}, \mathrm{Ca}^{++}$or $\mathrm{Mg}^{++}$, as well as enabling inhibition studies. ${ }^{91,209-211}$ Future 486 molecular dynamics simulations should extend the applicability of SIBFA to protein487 ligand binding.

488 Ren and coworkers have been systematically developing the AMOEBA protein force 489 field, and using it to study to several protein systems to understand protein-ligand 490 binding. ${ }^{57-59,61}$ More recently an X-Pol force field for proteins has been developed and 491 demonstrated in a simulation of solvated trypsin. ${ }^{32}$

492 The first attempt to compute the protein-ligand binding free energy using a polarizable 493 force field was made on the trypsin-benzamidine systems using AMOEBA. ${ }^{57,61,62}$ The 494 absolute binding free energy of benzamidine to trypsin, and the relative binding free 495 energies for a series of benzamidine analogs, were computed using a rigorous alchemical 496 transformation. AMOEBA was successful in evaluating the binding free energies 
497 accurately with an average error well within $1.0 \mathrm{kcal} / \mathrm{mol}$. A similar study on trypsin, 498 thrombin and urokinase was reported using another $a b$ initio QM-based polarizable force 499 field. ${ }^{212}$ A thermodynamic integration scheme was used to compute the relative binding 500 free energies, which were in excellent agreement with experimental data (root mean 501 squre error $(\mathrm{RMSE})=1.0 \mathrm{kcal} / \mathrm{mol})$

502 AMOEBA was later used to examine an ñentropic paradoxò associated with ligand 503 preorganization discovered in a previous study of conformationally constrained 504 phosphorylated-peptide analogs that bind to the SH2 domain of the growth receptor 505 binding protein $2(\mathrm{Grb} 2) .{ }^{59}$ The paradox refers to the unusual trend in which the binding 506 of unconstrained peptides (assumed to lose more entropy upon binding) is actually more 507 favorable entropically than are the constrained counterparts. AMOEBA correctly 508 reproduced the experimental trend and at the same time repeated a mechanism in which 509 the unconstrained peptide ligands were ñockedò by intramolecular nonbonded 510 interactions. The simulations uncovered a crucial caveat that had neen previously 511 acknowledged regarding the general design principle of ligand preorganization, which is 512 presumed by many to have a favorable effect on binding entropy.

513 More recently, Zhang et al. demonstrated the ability of AMOEBA in dealing with 514 systems with a metal ion. ${ }^{58}$ Those authors studied the Zinc-containing matrix 515 metalloproteinases (MMPs) in a complex with an inhibitor where the coordination of $516 \mathrm{Zn}^{++}$was with organic compounds and protein side chains. Polarization was found to play 517 a key role in $\mathrm{Zn}^{++}$coordination geometry in MMP. In addition, the relative binding free 518 energies of selected inhibitors binding with MMP13 were found to be in excellent 519 agreement with experimental results. As with the previous trypsin study, it was found that 
520 binding affinities are likely to be overestimated when the polarization between ligands

521 and environments is ignored.

522 Having a more rigorous physical model for treating polarization, the ability to model

523 protein-ligand interactions has been improved significantly. Systems involving highly

524 charged species, like metal ions, can now be treated with confidence. This in turn,

525 provides tremendous opportunities for investifating important proteins for drug discovery

526 and for protein engineering.

\section{3.5. Lipids}

528 With the rapid development of computational resources, simulations of large systems like

529 lipid bilayers with membrane proteins is feasible. ${ }^{126,213}$ Patel and coworkers have been 530 developing a polarizable force field for biomembranes to study the structure and 531 dynamics of ion channel systems. ${ }^{40}, 113,128,214$ Simulations of solvated DMPC 532 (dimyristoyl phosphatidylcholine) and dipalmitoylphosphatidylcholine (DPPC) bilayers 533 were reported. ${ }^{113,214}$ The distribution of the membrane components along the lipid bilayer 534 is similar to that from a fixed charge model. The water dipole moment was found to 535 increase from about 1.9 Debye in the middle of the membrane plane to the average bulk 536 value of 2.5 2.6 Debye. The lipid surface computed with the polarizable force field was 537 not improved from those of non-polarizable ones however. In addition, ion permeation in 538 a gramicidin A channel embedded in a DMPC bilayer was investigated. ${ }^{113}$ Davis and 539 Patel concluded that including the electronic polarization lowered the ion permeation free 540 energy barrier significantly, from $12 \mathrm{kcal} / \mathrm{mol}$ to $6 \mathrm{kcal} / \mathrm{mol}$.

\section{3.6. Continuum Solvents for Polarizable Biomolecular Solutes}


542 A continuum solvent replaces explicit atomic details with a bulk, mean-field response. It

543 is possible to demonstrate from statistical mechanics that an implicit solvent potential of

544 mean force (PMF) exists, wihch preserves exactly the solute thermodynamic properties

545 obtained from explicit solvent. ${ }^{215}$ It is possible to formulate a perfect implicit solvent in

546 principle, but in practice approximations are necessary to achieve efficiency. This

547 remains an active area of research. ${ }^{216}$ An implicit solvent PMF can be formulated via a

548 thermodynamic cycle that discharges the solute in vapor, grows the uncharged (apolar)

549 solute into a solvent $W_{\text {apolar }}(\mathbf{X})$ and finally recharges the solute within a continuum

550 dielectric $W_{\text {elec }}(\mathbf{X})$

$$
W_{\text {PMP }}(X)=W_{\text {apolar }}(X)+W_{\text {elec }}(X)
$$

552 The continuum electrostatic energy, including mobile electrolytes, can be described by 553 either the nonlinear Poisson-Boltzmann Equation (NPBE) or the simplified linearized 554 Poisson-Boltzmann Equation (LPBE)

$$
\nabla \cdot[\varepsilon(\mathbf{r}) \nabla \phi(\mathbf{r})]-\bar{\kappa}^{2}(\mathbf{r}) \phi(\mathbf{r})=-4 \pi \rho(\mathbf{r})
$$

556 where the coefficients are a function of position $\mathbf{r}, \phi$ is the potential, $\varepsilon$ is the pemittivity,

$557 \bar{\kappa}$ is the modified Debye-Hückel screening factor, and $\rho$ is the solute charge density. ${ }^{217}$, 558218 Implementations of a Poisson-Boltzmann continuum for many-body quantum 559 mechanical potentials have been applied to small molecules for decades. Examples 560 include the Polarizable Continuum Model (PCM) ${ }^{219,220}$, COSMO $^{221}$ and the Solvent 561 Model series (SMx). ${ }^{222}$ In contrast, applications of biomolecular continuum electrostatics 562 have been limited mainly to fixed partial charge solute descriptions for reasons of 563 computing efficiency force field availability. However, as a result of increasing 
577 continuum electrostatics (discussed next), which are fast enough to allow binding

578 affinities to be computed using alchemical sampling, rather than merely relying on end-

579 states. A key advantage of EPIC is that the biomolecular self-consistent field (SCF) is 580 determined by a single numerical finite-difference (FD) solution of the PBE, unlike the 581 aforementioned atom-centered PFF and PMPB models that require a new solution for

584 (i.e., the permanent field due to 1-2 or 1-3 interactions cannot be neglected). Although

computational power and the completion of the polarizable force fields for biomolecules described above, the coupling of classical many-body potentials to continuum electrostatics is now possible.

An important initial demonstration of polarizable biomolecules within a PoissonBoltzmann continuum used the Polarizable Force Field (PFF) of Maple et al. to model protein-ligand interactions. ${ }^{223}$ A second demonstration used the Electronic Polarization from Internal Continuum (EPIC), which accounts for intramolecular polarization using a continuum dielectric. ${ }^{224,225}$ Finally, the polarizable multipole Poisson-Boltzmann (PMPB) model based on the AMOEBA force field demonstrated that the self-consistent reaction field (SCRF) of proteins within a continuum solvent is consonant with the ensemble average response of explicit solvent. ${ }^{71}$ Contrarily, end-state calculations of protein-ligand binding affinity using the PMPB model were shown to not recapitulate explicit solvent alchemical free energies to chemical accuracy. ${ }^{61}$ This motivates development of analytic each SCF iteration. However, a tradeoff of EPICôs efficiency gain is a reduction in model flexibility because electrostatic masking rules cannot be incorporated into the FD solver masking of short-range bonded interactions is the standard approach used by essentially all biomolecular force fields, this is not possible for an EPIC style energy model. 
587 The first example of an analytic continuum electrostatic model for polarizable 588 biomolecules is the generalized Kirkwood (GK) model for the AMOEBA force field. ${ }^{73}$ 589 The AMOEBA/GK approach has been combined with alchemical sampling to predict 590 trypsin-ligand binding affinity with a correlation coefficient of 0.93 . This is a significant

591 improvement over the PMPB end-state approach. ${ }^{226}$ A second example, based on the 592 ABEEM $\sigma \pi$ fluctuating charge force field combined with a generalized Born (GB) 593 continuum electrostatic model, showed promising results for the computation of solvation 594 free energies for small organic molecules and peptide fragments. ${ }^{227}$

\section{3.7. Macromolecular X-ray Crystallography Refinement}

596 X-ray crystallography is the dominant experimental method for determining the 3597 dimensional coordinates of macromolecules. Collected diffraction data is the Fourier 598 transform of the ensemble average electron density of the macromolecular crystal. While 599 reciprocal space amplitudes of Bragg diffraction peaks are measured, their phases are not. 600 Instead, phase information is derived from the Fourier transform of a model structure that 601 is sufficiently close to the actual experimental ensemble. This is known as molecular 602 replacement (MR). After an initial model has been built into the electron density, further 603 refinement is based optimizating a target function $E_{\text {target }}$ of the form

$$
E_{\text {target }}^{*}=w_{A} E_{\mathrm{X}-\text { ray }}+E_{\text {Force Field }}^{*}
$$

605 where $E_{\mathrm{X} \text {-ray }}$ evaluates the agreement between measured and calculated diffraction 606 amplitudes, $E_{\text {Force Fisld }}$ restrains the model using prior knowledge of intra- and 607 intermolecular chemical forces and $w_{A}$ weights the relative strength of the two terms. ${ }^{77}$, 
$608{ }^{228}$ We now focus on the evolution of the prior chemical knowledge used during the X609 ray refinement process, and we culminate in ongoing work using polarizable force fields 610 in combination with PME electrostatics algorithms to obtain the most accurate, 611 informative biomolecular models possible.

612 The first application of molecular mechanics to macromolecular X-ray crystallography 613 refinement (based on fixed partial charge electrostatics evaluated using a spherical cutoff) 614 was on influenza-virus hemagglutinin by Weis et al. in $1990 .{ }^{229}$ This work demonstrated 615 that electrostatics maintained chemically reasonable hydrogen-bonding, although charged 616 surface residues were sometimes observed to form incorrect salt bridges. ${ }^{229}$ The latter 617 observation highlights the importance of accounting for dielectric screening arising from 618 the heterogeneous distribution of solvent within a macromolecular crystal, by using one 619 of the above described continuum electrostatics models. For example, the generalized 620 Born (GB) model for fixed charge electrostatics has been described, albeit with a 621 spherical cutoff approximation. ${ }^{230}$ Comparing refinements with and without GB 622 screening showed that roughly $10 \%$ of the amino acid side-chain conformations were 623 altered, with $75 \%$ of these side-chain differences due to residues at the macromolecular 624 surface. ${ }^{230}$ Although these first applications of fixed charge force field electrostatics were 625 encouraging, the use of spherical cutoffs to approximate crystal lattice sums is now 626 known to be only conditionally convergent and therefore prone to a variety of artifacts. ${ }^{231}$

627 In 1921, Ewald introduced an absolutely convergent solution to the problem of evaluating 628 electrostatic lattice summations in crystals. He did this by separating the problem into a 629 short-ranged real space sum and a periodic, smoothly varying, long-range sum that can be 630 evaluated efficiently in reciprocal space. ${ }^{232}$ This approach, now known as Ewald 
631 summation, has been described for both fixed partial charges and atomic multipoles. ${ }^{233 \text {, }}$

$632{ }^{234}$ More recently, the efficiency of Ewald summation was improved via the particle-mesh

633 Ewald (PME) algorithm, wherein the reciprocal space summation leverages the fast

634 Fourier transforms (FFT) $)^{235}$ via b-Spline interpolation ${ }^{236}$ for both fixed partial charge and

635 atomic multipole descriptions. ${ }^{237}$

636 The speed of the PME algorithm has been further improved for crystals by incorporating

637 explicit support for space group symmetry and by parallelization for heterogeneous

638 computer architectures. ${ }^{63}$ Combining the polarizable AMOEBA force field with

639 electrostatics evaluated using PME has been shown to improve macromolecular models

640 from X-ray crystallography refinement in a variety of contexts. ${ }^{64,77,238-240}$ At high

641 resolution $(\sim 1 \AA$ or lower $)$, the information contained within a polarizable atomic

642 multipole force field can be used to formulate the electron density of the scattering model

$643\left(E_{\mathrm{X} \text {-ray }}\right)$, in addition to contributing chemical restraints $\left(E_{\text {Force Fleld }}\right){ }^{64,238}$ The importance

644 of the prior chemical information contained in a polarizable force field is most significant

645 when positioning parts of the model that are not discernable from the experimental

646 electron density, as in the orientation of water hydrogen atoms ${ }^{239}$ or secondary structure

647 elements for mid-to-low resolution data sets $(\sim 3-4 \AA) .{ }^{63}$

648 Let use consider an example, the AMOEBA-assisted biomolecular X-ray refinement with 649 electrostatics evaluated via PME in the program Force Field X. This program was used to 650 re-refine nine mouse and human DNA methyltransferase 1 (Dnmt1) data sets deposited in 651 the Protein databank (PDB). Significant improvements in model quality (presented in 652 Table 1) were achieved as assayed by the MolProbity ${ }^{241}$ structure validation tool. The 653 MolProbity score is calibrated to reflect the expected resolution of the X-ray data. After 
661

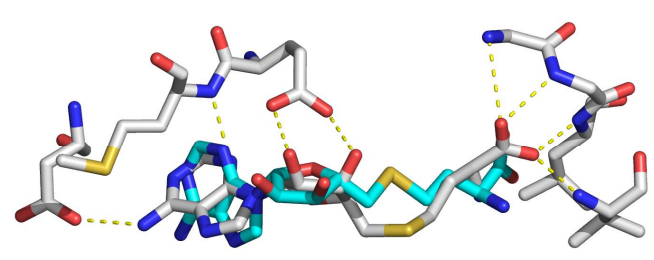
afterwards. Refinement with the Program Force Field X.

re-refinement, the average MolProbity score was reduced to 2.14 , indicating a level of model improvement consistent with collecting data to $0.67 \AA$ higher resolution. For example, the pose of $S$-adenosyl-L-homocysteine (SAH) from mouse (3PT6) and human (3PTA) structures differed by an RMSD of $1.6 \AA$ before re-refinement, but only $0.9 \AA$

Table 1. DNA Methyltransferase 1 (Dnmt1) Models Before and After Polarizable X-Ray

\begin{tabular}{|c|c|c|c|c|c|c|c|c|}
\hline \multirow[b]{3}{*}{ PDB } & \multirow[b]{3}{*}{ Res. $(\AA)$} & \multicolumn{3}{|c|}{ Protein Databank } & \multicolumn{4}{|c|}{ Re-Refined with Force Field $X$} \\
\hline & & \multicolumn{2}{|c|}{ Statistics } & MolProbity & \multicolumn{2}{|c|}{ Statistics } & \multicolumn{2}{|c|}{ MolProbity } \\
\hline & & $\mathrm{R}$ & $\mathrm{R}_{\text {free }}$ & Score $(\%)$ & $\mathrm{R}$ & $\mathrm{R}_{\text {free }}$ & Score & $(\%)$ \\
\hline $3 \mathrm{AV} 4$ & 2.8 & 0.232 & 0.267 & $2.87 \quad 68.0$ & 0.238 & - & 2.25 & 95.0 \\
\hline & & 188 & & 3.0979 .0 & 0.216 & & & 97.0 \\
\hline 3 & 3.1 & 0.195 & 0 & 2.9981 .0 & 0.213 & & 37 & 97.0 \\
\hline $3 \mathrm{EI}$ & & 0.213 & 0.2 & $2.27 \quad 78.0$ & 0.254 & & 2.09 & 87.0 \\
\hline & & 0.211 & 0.2 & 2.0154 .0 & 0. & & 77 & 74.0 \\
\hline & & 0 & & 2.9578 & 0.2 & & 7 & 99.0 \\
\hline & & 0.196 & 0. & 2.7260 .0 & 0.181 & & 1.90 & 97.0 \\
\hline & 3 & 0.257 & 0 & 3.6557 .0 & 0.211 & & .41 & 99.0 \\
\hline 3SWR & 2.5 & 0.220 & 0.272 & 2.6962 .0 & 0.204 & 0.264 & 2.03 & 95.0 \\
\hline Mean & 2.7 & 0.214 & 0.264 & 2.8068 .6 & 0.212 & 0.264 & 2.14 & 93.3 \\
\hline \multicolumn{4}{|c|}{ Mean Improvement } & & & & 0.67 & 24.8 \\
\hline
\end{tabular}

662 Figure 1. Polarizable biomolecular X-ray refinement on two Dnmt1 data sets. The left 663 panel shows the deposited pose of SAH from data sets 3PT6 (mouse, grey) and 3PTA 664 (human, cyan) do not agree (coord. RMSD 1.6 ̊). In the right panel, the poses of SAH 665 from mouse and human structures are more consistent (coord. RMSD $0.9 \AA$ ) after Force 


\subsection{Prediction of Organic Crystal Structure, Thermodynamics and Solubility}

669 It was emphasized in 1998 that predicting crystal structures from chemical composition

670 remained a major unsolved challenge. ${ }^{242}$ Significant progress has been made since then to

671 address this challenge, as evidenced by successes of the $4^{\text {th }}$ and $5^{\text {th }}$ blind tests of crystal

672 structure prediction (CSP) organized by the Cambridge Crystallographic Data Center

673 (CCDC). ${ }^{243,244}$ Prediction of crystal structures is important in the pharmaceutical industry, 674 where extensive experimental screens are necessary to explore the range of stable 675 polymorphs a molecule may form. The unique three-dimensional molecular packing of 676 each polymorph determines its physical properties such as stability and bioavailability.

677 For this reason, both FDA approval and patent protection are awarded to a specific 678 crystal polymorph, rather than to the molecule itself. To illustrate this point, eight 679 companies have filed eleven patents on five possible crystal forms of the molecule 680 cefdinir. $^{245}$ Prediction of thermodynamically stable crystal structures from chemical composition requires a potential energy function capable of distinguishing between large numbers of structures that are closely spaced in thermodynamic stability. ${ }^{246,247}$ In this section, we restrict our focus to energy models that explicitly account for electronic polarization classically $^{65,248,} 249$ and neglect the more expensive electronic structure methods

686 sometimes used to (re)score favorable structures. ${ }^{250}$

687 The vast majority of CSP has been limited to using intermolecular potentials that lack 688 explicit inclusion of polarization, ${ }^{249,}{ }^{251}$ although its importance has become a topic of 
689 interest $^{35,252-254}$. Non-polarizable force fields, based on fixed partial charges or fixed 690 atomic multipoles, must implicitly account for the $20 \%$ to $40 \%$ of the lattice energy 691 attributable induction. ${ }^{249}$ On the other hand, polarizable models such as the AMOEBA 692 force field for organic molecules ${ }^{54,255}$ based on the Thole damping scheme ${ }^{45}$ and the 693 Williams-Stone-Misquitta (WSM) method ${ }^{256,257}$ for obtaining distributed polarizabilities 694 allow one to include polarization during CSP explicitly.

695 Beyond polarization, modeling the conformational flexibility and corresponding 696 intermolecular energetics of organic molecules via sampling methods such as molecular 697 dynamics is essential to predicting the thermodynamic properties of crystals. ${ }^{258}$ For 698 example, the structure, stability and solubility of $n$-alkylamide crystals, from acetamide 699 through octanamide, can be predicted by an alchemical sampling method to compute the 700 sublimation/deposition phase transition free energy. ${ }^{65}$

\section{4. Summary}

702 Significant progress has been made in the past decade in developing general-purpose 703 polarizable force fields. Polarizable force fields have exhibited success in disparate 704 research areas including ion solvation, protein-ligand interactions, ion channels and lipids, macromolecular structural refinement and so on. There remain plenty of challenges ahead. The importance of polarization still needs to be established systematically for a wide

707 range of biological systems. While polarizable force fields in principle have better 708 transferability than do non-polarizable force fields, they are also expected to also perform 709 better in a broader range of systems, making parameterization a more elaborate process. 710 In addition to polarization, treatment of other physical effects, including high-order 
711 permanent charge distributions interactions, short-range electrostatic penetration and 712 charge-transfer effects need further improvement to advance the overall quality of 713 classical electrostatic models. Because computational efficiency (including the need for 714 parallelization) has been a major barrier to the adoption of polarizable force fields, better 715 and more efficient algorithms are also required to advance the application of polarizable 716 force fields. A future area for advancement is to combine the polarizable force fields with 717 fixed-charge force fields in a multiscale fashion, as is done with QM/MM. Technically 718 this can be achieved straightforwardly but caution is needed to ensure the interactions 719 across the two resolutions are balanced.

720 Acknowledgement. The authors are grateful to the support provided by Robert A. Welch 721 Foundation (F-1691). 


\section{REFERENCES}

724 1. A. Warshel and M. Levitt, Theoretical Studies of Enzymic Reactions - Dielectric, 725 Electrostatic and Steric Stabilization of Carbonium-Ion in Reaction of Lysozyme. Journal of Molecular Biology, 1976. 103(2): p. 227-249.

727 2. J.A. Mccammon, B.R. Gelin, and M. Karplus, Dynamics of Folded Proteins. Nature, 1977. 267(5612): p. 585-590.

3. M.A. Spackman, The Use of the Promolecular Charge Density to Approximate the Penetration Contribution to Intermolecular Electrostatic Energies. Chemical Physics Letters, 2006. 418(1-3): p. 158-162.

4. D. Nachtigallova, P. Hobza, and V. Spirko, Assigning the Nh Stretches of the Guanine Tautomers Using Adiabatic Separation: $\operatorname{CCSD}(T)$ Benchmark Calculations. Journal of Physical Chemistry A, 2008. 112(9): p. 1854-1856.

5. W.D. Cornell, P. Cieplak, C.I. Bayly, I.R. Gould, K.M. Merz, D.M. Ferguson, D.C. Spellmeyer, T. Fox, J.W. Caldwell, and P.A. Kollman, A 2nd Generation Force-Field for the Simulation of Proteins, Nucleic-Acids, and Organic-Molecules. Journal of the American Chemical Society, 1995. 117(19): p. 5179-5197.

A.D. MacKerell, D. Bashford, M. Bellott, R.L. Dunbrack, J.D. Evanseck, M.J. Field, S. Fischer, J. Gao, H. Guo, S. Ha, D. Joseph-McCarthy, L. Kuchnir, K. Kuczera, F.T.K. Lau, C. Mattos, S. Michnick, T. Ngo, D.T. Nguyen, B. Prodhom, W.E. Reiher, B. Roux, M. Schlenkrich, J.C. Smith, R. Stote, J. Straub, M. Watanabe, J. Wiorkiewicz-Kuczera, D. Yin, and M. Karplus, All-Atom Empirical Potential for Molecular Modeling and Dynamics Studies of Proteins. Journal of Physical Chemistry B, 1998. 102(18): p. 3586-3616.

745 7. H. Valdes, K. Pluhackova, M. Pitonak, J. Rezac, and P. Hobza, Benchmark Database on Isolated Small Peptides Containing an Aromatic Side Chain: Comparison between Wave 
Function and Density Functional Theory Methods and Empirical Force Field. Physical Chemistry Chemical Physics, 2008. 10(19): p. 2747-2757.

749 8. W. Jorgensen, D.S. Maxwell, and J. Tirado-Rives, Development and Testing of the OPLS All-Atom Force Field on Conformational Energetics and Properties of Organic Liquids. Journal of the American Chemical Society, 1996. 118(45): p. 11225-11236.

9. J.W. Ponder and D.A. Case, Force Fields for Protein Simulations. Advances in Protein Chemistry, 2003. 66: p. 27-85.

10. J. Rezac, P. Jurecka, K.E. Riley, J. Cerny, H. Valdes, K. Pluhackova, K. Berka, T. Rezac, M. Pitonak, J. Vondrasek, and P. Hobza, Quantum Chemical Benchmark Energy and Geometry Database for Molecular Clusters and Complex Molecular Systems (Www.Begdb.Com): A Users Manual and Examples. Collection of Czechoslovak Chemical Communications, 2008. 73(10): p. 1261-1270.

11. J. Rezac, K.E. Riley, and P. Hobza, S66: A Well-Balanced Database of Benchmark Interaction Energies Relevant to Biomolecular Structures. Journal of Chemical Theory and Computation, 2011. 7(8): p. 2427-2438.

12. K. Berka, R. Laskowski, K.E. Riley, P. Hobza, and J.i. Vondrášek, Representative Amino Acid Side Chain Interactions in Proteins. A Comparison of Highly Accurate Correlated Ab Initio Quantum Chemical and Empirical Potential Procedures. Journal of Chemical Theory and Computation, 2009. 5(4): p. 982-992.

13. J.A. Barker, Statistical Mechanics of Interacting Dipoles. Proceedings of the Royal Society of London. Series A, Mathematical and Physical Sciences, 1953. 219(1138): p. 367-372.

14. J.W. Caldwell and P.A. Kollman, Structure and Properties of Neat Liquids Using Nonadditive Molecular Dynamics: Water, Methanol, and N-Methylacetamide. Journal of Physical Chemistry, 1995. 99: p. 6208-6219. 
771 15. P.Y. Ren and J.W. Ponder, Polarizable Atomic Multipole Water Model for Molecular Mechanics Simulation. Journal of Physical Chemistry B, 2003. 107(24): p. 5933-5947.

16. G.A. Kaminski, H.A. Stern, B.J. Berne, R.A. Friesner, Y.X.X. Cao, R.B. Murphy, R.H. Zhou, and T.A. Halgren, Development of a Polarizable Force Field for Proteins Via Ab Initio Quantum Chemistry: First Generation Model and Gas Phase Tests. Journal of Computational Chemistry, 2002. 23(16): p. 1515-1531.

17. R.A. Friesner, Modeling Polarization in Proteins and Protein-Ligand Complexes: Methods and Preliminary Results. Advances in Protein Chemistry and Structural Biology, 2006. 72: p. 79-104.

18. P.Y. Ren and J.W. Ponder, Consistent Treatment of Inter- and Intramolecular Polarization in Molecular Mechanics Calculations. Journal of Computational Chemistry, 2002. 23(16): p. $1497-1506$.

19. L.F. Molnar, X. He, B. Wang, and K.M. Merz, Jr., Further Analysis and Comparative Study of Intermolecular Interactions Using Dimers from the S22 Database. The Journal of Chemical Physics, 2009. 131(6): p. 065102.

20. P. Cieplak, J. Caldwell, and P. Kollman, Molecular Mechanical Models for Organic and Biological Systems Going Beyond the Atom Centered Two Body Additive Approximation: Aqueous Solution Free Energies of Methanol and N-Methyl Acetamide, Nucleic Acid Base, and Amide Hydrogen Bonding and Chloroform/Water Partition Coefficients of the Nucleic Acid Bases. Journal of Computational Chemistry, 2001. 22(10): p. 1048-1057.

21. Z.X. Wang, W. Zhang, C. Wu, H.X. Lei, P. Cieplak, and Y. Duan, Strike a Balance: Optimization of Backbone Torsion Parameters of AMBER Polarizable Force Field for Simulations of Proteins and Peptides ( $\mathrm{Vol} 27, \mathrm{Pg}$ 781, 2006). Journal of Computational Chemistry, 2006. 27(8): p. 994-994. 
795 22. A. Holt and G. Karlström, Inclusion of the Quadrupole Moment When Describing Polarization. The Effect of the Dipole-Quadrupole Polarizability. Journal of Computational Chemistry, 2008. 29(12): p. 2033-2038.

23. S. Moghaddam, C. Yang, M. Rekharsky, Y.H. Ko, K. Kim, Y. Inoue, and M.K. Gilson, New Ultrahigh Affinity Host-Guest Complexes of Cucurbit[7]uril with Bicyclo[2.2.2]octane and Adamantane Guests: Thermodynamic Analysis and Evaluation of M2 Affinity Calculations. Journal of the American Chemical Society, 2011. 133(10): p. 3570-81.

24. D.P. Geerke and W.F. van Gunsteren, Calculation of the Free Energy of Polarization: Quantifying the Effect of Explicitly Treating Electronic Polarization on the Transferability of Force-Field Parameters. Journal of Physical Chemistry B, 2007. 111(23): p. 6425-6436.

25. G. Lamoureux, E. Harder, I.V. Vorobyov, B. Roux, and A.D. MacKerell, A Polarizable Model of Water for Molecular Dynamics Simulations of Biomolecules. Chemical Physics Letters, 2006. 418(1-3): p. 245-249.

26. G. Lamoureux, A.D. MacKerell, and B. Roux, A Simple Polarizable Model of Water Based on Classical Drude Oscillators. Journal of Chemical Physics, 2003. 119(10): p. 5185-5197.

27. J.L. Banks, G.A. Kaminski, R.H. Zhou, D.T. Mainz, B.J. Berne, and R.A. Friesner, Parametrizing a Polarizable Force Field from Ab Initio Data. I. The Fluctuating Point Charge Model. Journal of Chemical Physics, 1999. 110(2): p. 741-754.

28. S. Patel and C.L. Brooks III, CHARMM Fluctuating Charge Force Field for Proteins: I Parameterization and Application to Bulk Organic Liquid Simulations. Journal of Computational Chemistry, 2004. 25(1): p. 1-15.

29. S. Patel, A.D. Mackerell, and C.L. Brooks III, CHARMM Fluctuating Charge Force Field for Proteins: II - Protein/Solvent Properties from Molecular Dynamics Simulations Using a 
Nonadditive Electrostatic Model. Journal of Computational Chemistry, 2004. 25(12): p. $1504-1514$

30. A.K. Rappe and W.A. Goddard, Charge Equilibration for Molecular-Dynamics Simulations. Journal of Physical Chemistry, 1991. 95(8): p. 3358-3363.

31. L. Song, J. Han, Y.L. Lin, W. Xie, and J. Gao, Explicit Polarization (X-Pol) Potential Using Ab Initio Molecular Orbital Theory and Density Functional Theory. Journal of Physical Chemistry A, 2009. 113(43): p. 11656-64.

32. W. Xie, M. Orozco, D.G. Truhlar, and J. Gao, X-Pol Potential: An Electronic StructureBased Force Field for Molecular Dynamics Simulation of a Solvated Protein in Water. Journal of Chemical Theory and Computation, 2009. 5(3): p. 459-467.

33. M.J.L. Mills and P.L.A. Popelier, Polarisable Multipolar Electrostatics from the Machine Learning Method Kriging: An Application to Alanine. Theoretical Chemistry Accounts, 2012. 131(3).

P. Cieplak, F.Y. Dupradeau, Y. Duan, and J.M. Wang, Polarization Effects in Molecular Mechanical Force Fields. Journal of Physics-Condensed Matter, 2009. 21(33): p. 333102333123. with Explicit Inclusion of Electronic Polarizability: Theory and Applications. Theoretical Chemistry Accounts, 2009. 124(1-2): p. 11-28. Mobley, D.S. Lambrecht, R.A. DiStasio, M. Head-Gordon, G.N.I. Clark, M.E. Johnson, and 
T. Head-Gordon, Current Status of the AMOEBA Polarizable Force Field. Journal of Physical Chemistry B, 2010. 114(8): p. 2549-2564.

38. A. Warshel, M. Kato, and A.V. Pisliakov, Polarizable Force Fields: History, Test Cases, and Prospects. Journal of Chemical Theory and Computation, 2007. 3(6): p. 2034-2045.

39. S.W. Rick and S.J. Stuart, Potentials and Algorithms for Incorporating Polarizability in Computer Simulations. Reviews in Computational Chemistry, ed. K.B. Lipkowitz and D.B. Boyd. Vol. 18. 2002, New York: Wiley-VCH. 89-146.

40. S. Patel, J.E. Davis, and B.A. Bauer, Exploring Ion Permeation Energetics in Gramicidin A Using Polarizable Charge Equilibration Force Fields. Journal of the American Chemical Society, 2009. 131(39): p. 13890-1.

41. J. Applequist, J.R. Carl, and K.-K. Fung, An Atom Dipole Interaction Model for Molecular Polarizability. Application to Polyatomic Molecules and Determination of Atom Polarizabilities. Journal of the American Chemical Society, 1972. 94(9): p. 2952-2960.

42. C.R. Le Sueur and A.J. Stone, Practical Schemes for Distributed Polarizabilities. Molecular Physics, 1993. 78(5): p. 1267-1291.

43. D.R. Garmer and W.J. Stevens, Transferability of Molecular Distributed Polarizabilities from a Simple Localized Orbital Based Method. Journal of Physical Chemistry, 1989. 93(25): p. 8263-8270.

44. W. Wang and R.D. Skeel, Fast Evaluation of Polarizable Forces. The Journal of Chemical Physics, 2005. 123(16): p. 164107.

45. B.T. Thole, Molecular Polarizabilities Calculated with a Modified Dipole Interaction. Chemical Physics, 1981. 59(3): p. 341-350. 
46. J.F. Truchon, A. Nicholls, R.I. Iftimie, B. Roux, and C.I. Bayly, Accurate Molecular Polarizabilities Based on Continuum Electrostatics. Journal of Chemical Theory and Computation, 2008. 4(9): p. 1480-1493.

47. D. Van Belle, M. Froeyen, G. Lippens, and S.J. Wodak, Molecular-Dynamics Simulation of Polarizable Water by an Extended Lagrangian Method. Molecular Physics, 1992. 77(2): p. 239-255.

48. A.J. Stone, Distributed Multipole Analysis, or How to Describe a Molecular Charge Distribution. Chemical Physics Letters, 1981. 83(2): p. 233-239.

49. A.J. Stone, Distributed Multipole Analysis: Methods and Applications. Molecular Physics, 1985. 56(5): p. 1047-1064.

50. A.J. Stone, Distributed Multipole Analysis: Stability for Large Basis Sets. Journal of Chemical Theory and Computation, 2005. 1(6): p. 1128-1132.

51. A. Grossfield, P.Y. Ren, and J.W. Ponder, Ion Solvation Thermodynamics from Simulation with a Polarizable Force Field. Journal of the American Chemical Society, 2003. 125(50): p. $15671-15682$.

52. D. Jiao, C. King, A. Grossfield, T.A. Darden, and P.Y. Ren, Simulation of $\mathrm{Ca}^{2+}$ and $\mathrm{Mg}^{2+}$ Solvation Using Polarizable Atomic Multipole Potential. Journal of Physical Chemistry B, 2006. 110(37): p. 18553-18559.

53. J.C. Wu, J.P. Piquemal, R. Chaudret, P. Reinhardt, and P.Y. Ren, Polarizable Molecular Dynamics Simulation of $\mathrm{Zn}(I I)$ in Water Using the AMOEBA Force Field. Journal of Chemical Theory and Computation, 2010. 6(7): p. 2059-2070.

54. P. Ren, C. Wu, and J.W. Ponder, Polarizable Atomic Multipole-Based Molecular Mechanics for Organic Molecules. Journal of Chemical Theory and Computation, 2011. 7(10): p. 3143-3161. 
55. Y. Shi, C. Wu, J.W. Ponder, and P. Ren, Multipole Electrostatics in Hydration Free Energy Calculations. Journal of Computational Chemistry, 2011. 32(5): p. 967-977.

56. J.L. Jiang, Y.B. Wu, Z.X. Wang, and C. Wu, Assessing the Performance of Popular Quantum Mechanics and Molecular Mechanics Methods and Revealing the SequenceDependent Energetic Features Using 100 Tetrapeptide Models. Journal of Chemical Theory and Computation, 2010. 6(4): p. 1199-1209.

57. D. Jiao, P.A. Golubkov, T.A. Darden, and P. Ren, Calculation of Protein-Ligand Binding Free Energy by Using a Polarizable Potential. Proceedings of the National Academy of Sciences of the United States of America, 2008. 105(17): p. 6290-6295.

58. J. Zhang, W. Yang, J.P. Piquemal, and P. Ren, Modeling Structural Coordination and Ligand Binding in Zinc Proteins with a Polarizable Potential. Journal of Chemical Theory and Computation, 2012. 8: p. 1314-1324.

59. Y. Shi, C.Z. Zhu, S.F. Martin, and P. Ren, Probing the Effect of Conformational Constraint on Phosphorylated Ligand Binding to an SH2 Domain Using Polarizable Force Field Simulations. Journal of Physical Chemistry B, 2012. 116(5): p. 1716-27.

60. W. Jiang and B. Roux, Free Energy Perturbation Hamiltonian Replica-Exchange Molecular Dynamics (FEP/H-REMD) for Absolute Ligand Binding Free Energy Calculations. Journal of Chemical Theory and Computation, 2010. 6(9): p. 2559-2565.

61. D. Jiao, J.J. Zhang, R.E. Duke, G.H. Li, M.J. Schnieders, and P.Y. Ren, Trypsin-Ligand Binding Free Energies from Explicit and Implicit Solvent Simulations with Polarizable Potential. Journal of Computational Chemistry, 2009. 30(11): p. 1701-1711.

62. Y. Shi, D. Jiao, M.J. Schnieders, and P. Ren, Trypsin-Ligand Binding Free Energy Calculation with AMOEBA. Engineering in Medicine and Biology Society (EMBC). EMBC Annual International Conference of the IEEE, 2009: p. 2328-2331. 
911 63. M.J. Schnieders, T.D. Fenn, and V.S. Pande, Polarizable Atomic Multipole X-Ray Refinement: Particle Mesh Ewald Electrostatics for Macromolecular Crystals. Journal of Chemical Theory and Computation, 2011. 7(4): p. 1141-1156.

914

64. M.J. Schnieders, T.D. Fenn, V.S. Pande, and A.T. Brunger, Polarizable Atomic Multipole XRay Refinement: Application to Peptide Crystals. Acta Crystallographica Section DBiological Crystallography, 2009. 65: p. 952-965.

65. M.J. Schnieders, J. Baltrusaitis, Y. Shi, G. Chattree, L. Zheng, W. Yang, and P. Ren, The Structure, Thermodynamics and Solubility of Organic Crystals from Simulation with a Polarizable Force Field. Journal of Chemical Theory and Computation, 2012. 8(5): p.

67. M.S. Friedrichs, P. Eastman, V. Vaidyanathan, M. Houston, S. Legrand, A.L. Beberg, D.L.

68. D.A. Case, T.E. Cheatham, 3rd, T. Darden, H. Gohlke, R. Luo, K.M. Merz, Jr., A. Onufriev, 1721-1736.

66. J.W. Ponder, Tinker: Software Tools for Molecular Design Washington University http://dasher.wustl.edu/tinker/, 2012. v6.0. Ensign, C.M. Bruns, and V.S. Pande, Accelerating Molecular Dynamic Simulation on Graphics Processing Units. Journal of Computational Chemistry, 2009. 30(6): p. 864-72. C. Simmerling, B. Wang, and R.J. Woods, The AMBER Biomolecular Simulation Programs. Journal of Computational Chemistry, 2005. 26(16): p. 1668-1688.

69. M.J. Schnieders, T.D. Fenn, J. Wu, W. Yang, and P. Ren, Force Field X Open Source, Platform Independent Modules for Molecular Biophsyics Simulations. http://ffx.kenai.com, 2011.

932 70. J.W. Ponder, Tinker: Software Tools for Molecular Design. Saint Louis, MO. p. TINKER: Software Tools for Molecular Design. http://dasher.wustl.edu, 2012. 
71. M.J. Schnieders, N.A. Baker, P.Y. Ren, and J.W. Ponder, Polarizable Atomic Multipole Solutes in a Poisson-Boltzmann Continuum. Journal of Chemical Physics, 2007. 126(12).

72. N.A. Baker, D. Sept, S. Joseph, M.J. Holst, and J.A. McCammon, Electrostatics of Nanosystems: Application to Microtubules and the Ribosome. Proceedings of the National Academy of Sciences at the United States of America, 2001. 98(18): p. 1003710041.

73. M.J. Schnieders and J.W. Ponder, Polarizable Atomic Multipole Solutes in a Generalized Kirkwood Continuum. Journal of Chemical Theory and Computation, 2007. 3(6): p. 20832097.

74. B.R. Brooks, C.L. Brooks III, A.D. MacKerell, Jr., L. Nilsson, R.J. Petrella, B. Roux, Y. Won, G. Archontis, C. Bartels, S. Boresch, A. Caflisch, L. Caves, Q. Cui, A.R. Dinner, M. Feig, S. Fischer, J. Gao, M. Hodoscek, W. Im, K. Kuczera, T. Lazaridis, J. Ma, V. Ovchinnikov, E. Paci, R.W. Pastor, C.B. Post, J.Z. Pu, M. Schaefer, B. Tidor, R.M. Venable, H.L. Woodcock, X. Wu, W. Yang, D.M. York, and M. Karplus, CHARMM: The Biomolecular Simulation Program. Journal of Computational Chemistry, 2009. 30(10): p. 1545-614.

75. H.L. Woodcock, B.T. Miller, M. Hodoscek, A. Okur, J.D. Larkin, J.W. Ponder, and B.R. Brooks, MSCALE: A General Utility for Multiscale Modeling. Journal of Chemical Theory and Computation, 2011. 7(4): p. 1208-1219.

76. L. Zheng, M. Chen, and W. Yang, Random Walk in Orthogonal Space to Achieve Efficient Free-Energy Simulation of Complex Systems. Proceedings of the National Academy of Sciences at the United States of America, 2008. 105(51): p. 20227-20232.

77. T.D. Fenn and M.J. Schnieders, Polarizable Atomic Multipole X-Ray Refinement: Weighting Schemes for Macromolecular Diffraction. Acta Crystallographica Section D, 2011. 67(11): p. 957-65. 
78. P. Eastman and V.S. Pande, Efficient Nonbonded Interactions for Molecular Dynamics on a Graphics Processing Unit. Journal of Computational Chemistry, 2010. 31(6): p. 12681272.

79. N. Gresh, P. Claverie, and A. Pullman, Theoretical Studies of Molecular Conformation Derivation of an Additive Procedure for the Computation of Intramolecular Interaction Energies - Comparison with ab Initio SCF Computations. Theoretica Chimica Acta, 1984. 66(1): p. 1-20.

80. N. Gresh, Energetics of $\mathrm{Zn}^{2+}$ Binding to a Series of Biologically Relevant Ligands - $A$ Molecular Mechanics Investigation Grounded on Ab-Initio Self-Consistent-Field Supermolecular Computations. Journal of Computational Chemistry, 1995. 16(7): p. 856882.

81. J.P. Piquemal, B. Williams-Hubbard, N. Fey, R.J. Deeth, N. Gresh, and C. Giessner-Prettre, Inclusion of the Ligand Field Contribution in a Polarizable Molecular Mechanics: SIBFA-LF. Journal of Computational Chemistry, 2003. 24(16): p. 1963-1970.

82. J.P. Piquemal, H. Chevreau, and N. Gresh, Toward a Separate Reproduction of the Contributions to the Hartree-Fock and DFT Intermolecular Interaction Energies by Polarizable Molecular Mechanics with the SIBFA Potential. Journal of Chemical Theory and Computation, 2007. 3(3): p. 824-837.

83. N. Gresh, G.A. Cisneros, T.A. Darden, and J.P. Piquemal, Anisotropic, Polarizable Molecular Mechanics Studies of Inter- and Intramolecular Interactions and LigandMacromolecule Complexes. A Bottom-up Strategy. Journal of Chemical Theory and Computation, 2007. 3(6): p. 1960-1986.

84. J.P. Piquemal, N. Gresh, and C. Giessner-Prettre, Improved Formulas for the Calculation of the Electrostatic Contribution to the Intermolecular Interaction Energy from 
Multipolar Expansion of the Electronic Distribution. Journal of Physical Chemistry A, 2003. 107(48): p. 10353-10359.

85. N. Gresh, P. Claverie, and A. Pullman, Intermolecular Interactions - Elaboration on an Additive Procedure Including an Explicit Charge-Transfer Contribution. International Journal of Quantum Chemistry, 1986. 29(1): p. 101-118.

86. N. Gresh, Inter- and Intramolecular Interactions. Inception and Refinements of the SIBFA, Molecular Mechanics (SMM) Procedure, A Separable, Polarizable Methodology Grounded on Ab Initio SCF/MP2 Computations. Examples of Applications to Molecular Recognition Problems. Journal De Chimie Physique Et De Physico-Chimie Biologique, 1997. 94(7-8): p. 1365-1416.

992 87. J. Antony, J.P. Piquemal, and N. Gresh, Complexes of Thiomandelate and Captopril Mercaptocarboxylate Inhibitors to Metallo-Beta-Lactamase by Polarizable Molecular Mechanics. Validation on Model Binding Sites by Quantum Chemistry. Journal of Computational Chemistry, 2005. 26(11): p. 1131-1147.

88. C. Roux, N. Gresh, L.E. Perera, J.P. Piquemal, and L. Salmon, Binding of 5-Phospho-DArabinonohydroxamate and 5-Phospho-D-Arabinonate Inhibitors to Zinc

89. L.M.M. Jenkins, T. Hara, S.R. Durell, R. Hayashi, J.K. Inman, J.P. Piquemal, N. Gresh, and E. Appella, Specificity of Acylf Transfer from 2-Mercaptobenzamide Thioesters to the HIV-1 Nucleocapsid Protein. Journal of the American Chemical Society, 2007. 129(36): p. 11067-11078. 
1005 90. J. Foret, B. de Courcy, N. Gresh, J.P. Piquemal, and L. Salmon, Synthesis and Evaluation of Non-Hydrolyzable D-Mannose 6-Phosphate Surrogates Reveal 6-Deoxy-6Dicarboxymethyl-D-Mannose as a New Strong Inhibitor of Phosphomannose Isomerases. Bioorganic \& Medicinal Chemistry, 2009. 17(20): p. 7100-7107.

91. N. Gresh, N. Audiffren, J.P. Piquemal, J. de Ruyck, M. Ledecq, and J. Wouters, Analysis of the Interactions Taking Place in the Recognition Site of a Bimetallic Mg(II)-Zn(II) Enzyme, Isopentenyl Diphosphate Isomerase. A Parallel Quantum-Chemical and Polarizable Molecular Mechanics Study. Journal of Physical Chemistry B, 2010. 114(14): p. 48844895.

92. C. Roux, F. Bhatt, J. Foret, B. de Courcy, N. Gresh, J.P. Piquemal, C.J. Jeffery, and L. Salmon, The Reaction Mechanism of Type I Phosphomannose Isomerases: New Information from Inhibition and Polarizable Molecular Mechanics Studies. ProteinsStructure Function and Bioinformatics, 2011. 79(1): p. 203-20.

1018 93. M. Ledecq, F. Lebon, F. Durant, C. Giessner-Prettre, A. Marquez, and N. Gresh, Modeling of Copper(II) Complexes with the SIBFA Polarizable Molecular Mechanics Procedure.

1022 94. A. Marjolin, C. Gourlaouen, C. Clavaguéra, P. Ren, J. Wu, N. Gresh, J.-P. Dognon, and J.-P. Application to a New Class of HIV-1 Protease Inhibitors. Journal of Physical Chemistry B, 2003. 107(38): p. 10640-10652. Piquemal, Toward Accurate Solvation Dynamics of Lanthanides and Actinides in Water Using Polarizable Force Fields: From Gas-Phase Energetics to Hydration Free Energies. Theoretical Chemistry Accounts: Theory, Computation, and Modeling (Theoretica Chimica Acta), 2012. 131(4): p. 1-14. 
95. F. Rogalewicz, G. Ohanessian, and N. Gresh, Interaction of Neutral and Zwitterionic Glycine with $\mathrm{Zn}^{2+}$ in Gas Phase: ab Initio and SIBFA Molecular Mechanics Calculations. Journal of Computational Chemistry, 2000. 21(11): p. 963-973.

96. N. Gresh, G. Tiraboschi, and D.R. Salahub, Conformational Properties of a Model Alanyl Dipeptide and of Alanine-Derived Oligopeptides: Effects of Solvation in Water and in Organic Solvents - A Combined SIBFA/Continuum Reaction Field, ab Initio Self-Consistent Field, and Density Functional Theory Investigation. Biopolymers, 1998. 45(6): p. 405-425.

97. J. Graf, P.H. Nguyen, G. Stock, and H. Schwalbe, Structure and Dynamics of the Homologous Series of Alanine Peptides: A Joint Molecular Dynamics/NMR Study. Journal of the American Chemical Society, 2007. 129(5): p. 1179-1189.

98. G.A. Cisneros, T.A. Darden, N. Gresh, J. Pilme, P. Reinhardt, O. Parisel, and J.P. Piquemal, Design of Next Generation Force Fields from ab Initio Computations: Beyond Point Charges Electrostatics, in Multi-scale Quantum Models for Biocatalysis, D.M. York and T.S. Lee, Editors. 2009, Springer Science.

99. J.P. Piquemal, G.A. Cisneros, P. Reinhardt, N. Gresh, and T.A. Darden, Towards a Force Field Based on Density Fitting. Journal of Chemical Physics, 2006. 124(10): p. 104101.

100. G.A. Cisneros, J.P. Piquemal, and T.A. Darden, Generalization of the Gaussian Electrostatic Model: Extension to Arbitrary Angular Momentum, Distributed Multipoles, and Speedup with Reciprocal Space Methods. J Chem Phys, 2006. 125(18): p. 184101.

101. S. Brdarski and G. Karlström, Modeling of the Exchange Repulsion Energy. Journal of Physical Chemistry A, 1998. 102(42): p. 8182-8192.

102. M.A. Carignano, G. Karlström, and P. Linse, Polarizable lons in Polarizable Water: A Molecular Dynamics Study. Journal of Physical Chemistry B, 1997. 101(7): p. 1142-1147. 
1050

1051

1052

1053

1054

1055

1056

1057

1058

1059

1060

1061

1062

1063

1064

1065

1066

1067

1068

1069

1070

1071

1072

1073

103. A. Holt and G. Karlström, Improvement of the NEMO Potential by Inclusion of Intramolecular Polarization. International Journal of Quantum Chemistry, 2009. 109(6): p. $1255-1266$.

104. L. Gagliardi, R. Lindh, and G. Karlström, Local Properties of Quantum Chemical Systems: The Loprop Approach. Journal of Chemical Physics, 2004. 121(10): p. 4494-4500.

105. J.M. Hermida-Ramon, S. Brdarski, G. Karlström, and U. Berg, Inter- and Intramolecular Potential for the N-Formylglycinamide-Water System. A Comparison between Theoretical Modeling and Empirical Force Fields. Journal of Computational Chemistry, 2003. 24(2): p. 161-176.

106. D. Hagberg, G. Karlström, B.O. Roos, and L. Gagliardi, The Coordination of Uranyl in Water: A Combined Quantum Chemical and Molecular Simulation Study. Journal of the American Chemical Society, 2005. 127(41): p. 14250-14256.

107. P.A. Kollman, I. Massova, C. Reyes, B. Kuhn, S.H. Huo, L. Chong, M. Lee, T. Lee, Y. Duan, W. Wang, O. Donini, P. Cieplak, J. Srinivasan, D.A. Case, and T.E. Cheatham, Calculating Structures and Free Energies of Complex Molecules: Combining Molecular Mechanics and Continuum Models. Accounts of Chemical Research, 2000. 33(12): p. 889-897.

108. P. Drude, C.R. Mann, and R.A. Millikan, The Theory of Optics. 1902, Longmans, Green, and Co., New York, p1-572.

109. E. Harder, V.M. Anisimov, T.W. Whitfield, A.D. MacKerell, and B. Roux, Understanding the Dielectric Properties of Liquid Amides from a Polarizable Force Field. Journal of Physical Chemistry B, 2008. 112(11): p. 3509-3521.

110. R.O. Dror, R.M. Dirks, J.P. Grossman, H. Xu, and D.E. Shaw, Biomolecular Simulation: A Computational Microscope for Molecular Biology. Annual Review of Biophysics, 2012. 41(1): p. 429-452. 
111. E.M. Myshakin, H. Jiang, and K.D. Jordan, Phys 549-Molecular Dynamics Simulations of Methane Hydrate Decomposition Using a Polarizable Force Field. Abstracts of Papers of the American Chemical Society, 2007. 234.

112. P.E.M. Lopes, G. Lamoureux, B. Roux, and A.D. MacKerell, Polarizable Empirical Force Field for Aromatic Compounds Based on the Classical Drude Oscillator. Journal of Physical Chemistry B, 2007. 111(11): p. 2873-2885.

113. J.E. Davis and S. Patel, Charge Equilibration Force Fields for Lipid Environments: Applications to Fully Hydrated DPPC Bilayers and DMPC-Embedded Gramicidin A. Journal of Physical Chemistry B, 2009. 113(27): p. 9183-9196.

114. R. Xiong, X.M. Cai, J. Wei, and P.Y. Ren, Some Insights into the Binding Mechanism of Aurora B Kinase Gained by Molecular Dynamics Simulation. Journal of Molecular Modeling, 2012: p. 3049-3060.

115. T.S. Kaoud, H. Park, S. Mitra, C. Yan, C.C. Tseng, Y. Shi, J. Jose, J.M. Taliaferro, K. Lee, P. Ren, J. Hong, and K.N. Dalby, Manipulating JNK Signaling with (-)-Zuonin A. ACS chemical biology, 2012: p1873-1883.

116. G.L. Warren and S. Patel, Hydration Free Energies of Monovalent lons in Transferable Intermolecular Potential Four Point Fluctuating Charge Water: An Assessment of Simulation Methodology and Force Field Performance and Transferability. Journal of Chemical Physics, 2007. 127(6): p. 64509-64528.

117. C.M. Baker, P.E. Lopes, X. Zhu, B. Roux, and A.D. MacKerell, Jr., Accurate Calculation of Hydration Free Energies Using Pair-Specific Lennard-Jones Parameters in the CHARMM Drude Polarizable Force Field. Journal of Chemical Theory and Computation, 2010. 6(4): p. $1181-1198$. 
118. J.M. Wang, R.M. Wolf, J.W. Caldwell, P.A. Kollman, and D.A. Case, Development and Testing of a General AMBER Force Field. Journal of Computational Chemistry, 2004. 25(9): p. 1157-1174.

119. T.W. Whitfield, S. Varma, E. Harder, G. Lamoureux, S.B. Rempe, and B. Roux, Theoretical Study of Aqueous Solvation of $\mathrm{K}(+)$ Comparing Ab Initio, Polarizable, and Fixed-Charge Models. Journal of Chemical Theory and Computation, 2007. 3(6): p. 2068-2082.

120. H.B. Yu, T.W. Whitfield, E. Harder, G. Lamoureux, I. Vorobyov, V.M. Anisimov, A.D. MacKerell, and B. Roux, Simulating Monovalent and Divalent lons in Aqueous Solution Using a Drude Polarizable Force Field. Journal of Chemical Theory and Computation, 2010. 6(3): p. 774-786.

121. Z.Y. Lu and Y.K. Zhang, Interfacing Ab Initio Quantum Mechanical Method with Classical Drude Osillator Polarizable Model for Molecular Dynamics Simulation of Chemical Reactions. Journal of Chemical Theory and Computation, 2008. 4(8): p. 1237-1248.

122. B.R. Brooks, R.E. Bruccoeri, B.D. Olafson, D.J. States, S. Swaminathan, and M. Karplus, CHARMM: A Program for Macromolecular Energy, Minimization, and Dynamics Calculations. Journal of Computational Chemistry, 1983. 4(2): p. 187-217.

123. J.C. Phillips, R. Braun, W. Wang, J. Gumbart, E. Tajkhorshid, E. Villa, C. Chipot, R.D. Skeel, L. Kale, and K. Schulten, Scalable Molecular Dynamics with NAMD. Journal of Computational Chemistry, 2005. 26(16): p. 1781-802.

124. W. Jiang, D.J. Hardy, J.C. Phillips, A.D. Mackerell, K. Schulten, and B. Roux, HighPerformance Scalable Molecular Dynamics Simulations of a Polarizable Force Field Based on Classical Drude Oscillators in NAMD. Journal of Physical Chemistry Letters, 2011. 2(2): p. 87-92. 
125. T.S. Kaoud, C. Yan, S. Mitra, C.-C. Tseng, J. Jose, J.M. Taliaferro, M. Tuohetahuntila, A. Devkota, R. Sammons, J. Park, H. Park, Y. Shi, J. Hong, P. Ren, and K.N. Dalby, From in Silico Discovery to Intracellular Activity: Targeting JNK-Protein Interactions with Small Molecules. ACS Medicinal Chemistry Letters, 2012. 3(9): p. 721-725.

126. J.D. Chodera, W.C. Swope, F. Noe, J.H. Prinz, M.R. Shirts, and V.S. Pande, Dynamical Reweighting: Improved Estimates of Dynamical Properties from Simulations at Multiple Temperatures. The Journal of Chemical Physics, 2011. 134(24): p. 244107.

127. M.R. Shirts, D.L. Mobley, J.D. Chodera, and V.S. Pande, Accurate and Efficient Corrections for Missing Dispersion Interactions in Molecular Simulations. Journal of Physical Chemistry B, 2007. 111(45): p. 13052-63.

128. B.A. Bauer, T.R. Lucas, D.J. Meninger, and S. Patel, Water Permeation through DMPC Lipid Bilayers Using Polarizable Charge Equilibration Force Fields. Chemical Physics Letters, 2011. 508(4-6): p. 289-294.

129. B.A. Bauer, G.L. Warren, and S. Patel, Incorporating Phase-Dependent Polarizability in Nonadditive Electrostatic Models for Molecular Dynamics Simulations of the Aqueous Liquid-Vapor Interface. Journal of Chemical Theory and Computation, 2009. 5(2): p. 359373.

130. W. Xie and J. Gao, The Design of a Next Generation Force Field: The X-Pol Potential. Journal of Chemical Theory and Computation, 2007. 3(6): p. 1890-1900.

131. J.L. Gao, A Molecular-Orbital Derived Polarization Potential for Liquid Water. Journal of Chemical Physics, 1998. 109(6): p. 2346-2354.

132. S.J. Wierzchowski, D.A. Kofke, and J.L. Gao, Hydrogen Fluoride Phase Behavior and Molecular Structure: A QM/MM Potential Model Approach. Journal of Chemical Physics, 2003. 119(14): p. 7365-7371. 
133. W.S. Xie, L.C. Song, D.G. Truhlar, and J.L. Gao, Incorporation of a QM/MM Buffer Zone in the Variational Double Self-Consistent Field Method. Journal of Physical Chemistry B, 2008. 112(45): p. 14124-14131.

134. W.S. Xie, L.C. Song, D.G. Truhlar, and J.L. Gao, The Variational Explicit Polarization Potential and Analytical First Derivative of Energy: Towards a Next Generation Force Field. Journal of Chemical Physics, 2008. 128(23): p234108.

135. Y.J. Wang, C.P. Sosa, A. Cembran, D.G. Truhlar, and J.L. Gao, Multilevel X-Pol: A Fragment-Based Method with Mixed Quantum Mechanical Representations of Different Fragments. Journal of Physical Chemistry B, 2012. 116(23): p. 6781-6788.

136. G.A. Kaminski, R.A. Friesner, and R.H. Zhou, A Computationally Inexpensive Modification of the Point Dipole Electrostatic Polarization Model for Molecular Simulations. Journal of Computational Chemistry, 2003. 24(3): p. 267-276.

137. G.A. Kaminski, H.A. Stern, B.J. Berne, and R.A. Friesner, Development of an Accurate and Robust Polarizable Molecular Mechanics Force Field from Ab Initio Quantum Chemistry. Journal of Physical Chemistry A, 2004. 108(4): p. 621-627.

138. B.C. Kim, T. Young, E. Harder, R.A. Friesner, and B.J. Berne, Structure and Dynamics of the Solvation of Bovine Pancreatic Trypsin Inhibitor in Explicit Water: A Comparative Study of the Effects of Solvent and Protein Polarizability. Journal of Physical Chemistry B, 2005. 109(34): p. 16529-16538.

139. G.A. Kaminski, S.Y. Ponomarev, and A.B. Liu, Polarizable Simulations with Second Order Interaction Model - Force Field and Software for Fast Polarizable Calculations: Parameters for Small Model Systems and Free Energy Calculations. Journal of Chemical Theory and Computation, 2009. 5(11): p. 2935-2943. 
140. S.Y. Ponomarev and G.A. Kaminski, Polarizable Simulations with Second Order Interaction Model (POSSIM) Force Field: Developing Parameters for Alanine Peptides and Protein Backbone. Journal of Chemical Theory and Computation, 2011. 7(5): p. 14151427.

141. S.Y. Ponomarev and G. Kaminski, Polarizable Force Field for Protein Simulations POSSIM: Alanine Dipeptide and Tetrapeptide Parameters, and Stability of the Alanine 13 AlphaHelix in Water. Abstracts of Papers of the American Chemical Society, 2011. 241.

142. O. Borodin, R. Douglas, G.A. Smith, F. Trouw, and S. Petrucci, MD Simulations and Experimental Study of Structure, Dynamics, and Thermodynamics of Poly(Ethylene Oxide) and Its Oligomers. Journal of Physical Chemistry B, 2003. 107(28): p. 6813-6823.

143. O. Borodin and G.D. Smith, Development of Quantum Chemistry-Based Force Fields for Poly(Ethylene Oxide) with Many-Body Polarization Interactions. Journal of Physical Chemistry B, 2003. 107(28): p. 6801-6812.

144. O. Borodin, G.D. Smith, and R. Douglas, Force Field Development and MD Simulations of Poly(Ethylene Oxide)/LIBF 4 Polymer Electrolytes. Journal of Physical Chemistry B, 2003. 107(28): p. 6824-6837.

145. P. Paricaud, M. Predota, A.A. Chialvo, and P.T. Cummings, From Dimer to Condensed Phases at Extreme Conditions: Accurate Predictions of the Properties of Water by a Gaussian Charge Polarizable Model. Journal of Chemical Physics, 2005. 122(24): p244511.

146. J.L. Rivera, F.W. Starr, P. Paricaud, and P.T. Cummings, Polarizable Contributions to the Surface Tension of Liquid Water. Journal of Chemical Physics, 2006. 125(9): p094712.

147. Z. Tao and P.T. Cummings, Molecular Dynamics Simulation of Inorganic Ions in POE Aqueous Solution. Molecular Simulation, 2007. 33(15): p. 1255-1260. 
148. O. Borodin, Polarizable Force Field Development and Molecular Dynamics Simulations of Ionic Liquids. The Journal of Physical Chemistry B, 2009. 113(33): p. 11463-11478.

149. F.H. Stillinger and C.W. David, Polarization Model for Water and Its Ionic Dissociation Products. Journal of Chemical Physics, 1978. 69(4): p. 1473-1484.

150. B. Guillot, A Reappraisal of What We Have Learnt During Three Decades of Computer Simulations of Water. Journal of Molecular Liquids, 2002. 101(1-3): p. 219-260.

151. P. Ahlström, A. Wallqvist, S. Engström, and B. Jonsson, A Molecular-Dynamics Study of Polarizable Water. Molecular Physics, 1989. 68(3): p. 563-581.

152. M. Sprik and M.L. Klein, A Polarizable Model for Water Using Distributed Charge Sites. Journal of Chemical Physics, 1988. 89(12): p. 7556-7560.

153. P. Cieplak, P. Kollman, and T. Lybrand, A New Water Potential Including Polarization Application to Gas-Phase, Liquid, and Crystal Properties of Water. Journal of Chemical Physics, 1990. 92(11): p. 6755-6760.

154. U. Niesar, G. Corongiu, E. Clementi, G.R. Kneller, and D.K. Bhattacharya, MolecularDynamics Simulations of Liquid Water Using the NCC Ab Initio Potential. Journal of Physical Chemistry, 1990. 94(20): p. 7949-7956.

155. L.X. Dang, Development of Nonadditive Intermolecular Potentials Using MolecularDynamics - Solvation of $\mathrm{Li}^{+}$and F lons in Polarizable Water. Journal of Chemical Physics, 1992. 96(9): p. 6970-6977.

156. L.X. Dang and T.M. Chang, Molecular Dynamics Study of Water Clusters, Liquid, and Liquid-Vapor Interface of Water with Many-Body Potentials. Journal of Chemical Physics, 1997. 106(19): p. 8149-8159.

157. C.J. Burnham and S.S. Xantheas, Development of Transferable Interaction Models for Water. III. A Flexible, All-Atom Polarizable Potential (TTM2-F) Based on Geometry 
Dependent Charges Derived from an Ab Initio Monomer Dipole Moment Surface. Journal of Chemical Physics, 2002. 116(12): p. 5115-5124.

158. C.J. Burnham and S.S. Xantheas, Development of Transferable Interaction Models for Water. I. Prominent Features of the Water Dimer Potential Energy Surface. Journal of Chemical Physics, 2002. 116(4): p. 1479-1492.

159. C.J. Burnham and S.S. Xantheas, Development of Transferable Interaction Models for Water. lii. Reparametrization of an All-Atom Polarizable Rigid Model (Ttm2-R) from First Principles. Journal of Chemical Physics, 2002. 116(4): p. 1500-1510.

160. S.S. Xantheas, C.J. Burnham, and R.J. Harrison, Development of Transferable Interaction Models for Water. II. Accurate Energetics of the First Few Water Clusters from First Principles. Journal of Chemical Physics, 2002. 116(4): p. 1493-1499.

161. H.B. Yu, T. Hansson, and W.F. van Gunsteren, Development of a Simple, Self-Consistent Polarizable Model for Liquid Water. Journal of Chemical Physics, 2003. 118(1): p. 221234.

162. H.B. Yu and W.F. van Gunsteren, Charge-on-Spring Polarizable Water Models Revisited: From Water Clusters to Liquid Water to Ice. Journal of Chemical Physics, 2004. 121(19):

163. S.W. Rick, S.J. Stuart, and B.J. Berne, Dynamical Fluctuating Charge Force-Fields Application to Liquid Water. Journal of Chemical Physics, 1994. 101(7): p. 6141-6156.

164. H.A. Stern, F. Rittner, B.J. Berne, and R.A. Friesner, Combined Fluctuating Charge and Polarizable Dipole Models: Application to a Five-Site Water Potential Function. Journal of Chemical Physics, 2001. 115(5): p. 2237-2251. 
General-Purpose Quantum Mechanical Polarizable Force Field. Proceedings of the National Academy of Sciences of the United States of America, 2006. 103(23): p. 86138617.

166. J.M. Wang, P. Cieplak, and P.A. Kollman, How Well Does a Restrained Electrostatic Potential (RESP) Model Perform in Calculating Conformational Energies of Organic and Biological Molecules? Journal of Computational Chemistry, 2000. 21(12): p. 1049-1074.

167. J. Jeon, A.E. Lefohn, and G.A. Voth, An Improved Polarflex Water Model. Journal of Chemical Physics, 2003. 118(16): p. 7504-7518.

1247 168. M.K. Gilson and H.X. Zhou, Calculation of Protein-Ligand Binding Affinities. Annual Review of Biophysics and Biomolecular Structure, 2007. 36: p. 21-42.

1249 169. A.J. Lee and S.W. Rick, The Effects of Charge Transfer on the Properties of Liquid Water. 1250 Journal of Chemical Physics, 2011. 134(18): p. 184507.

1251 170. E. Muchova, I. Gladich, S. Picaud, P.N.M. Hoang, and M. Roeselova, The Ice-Vapor 1252 Interface and the Melting Point of Ice I-H for the Polarizable POL3 Water Model. Journal of Physical Chemistry A, 2011. 115(23): p. 5973-5982.

1254 171. B.A. Bauer and S. Patel, Properties of Water Along the Liquid-Vapor Coexistence Curve Via Molecular Dynamics Simulations Using the Polarizable TIP4P-QDP-LJ Water Model. The Journal of Chemical Physics, 2009. 131(8): p. 084709.

1257 172. T.P. Lybrand and P.A. Kollman, Water-Water and Water-Ion Potential Functions Including Terms for Many-Body Effects. Journal of Chemical Physics, 1985. 83(6): p. 2923-2933.

173. S.J. Stuart and B.J. Berne, Effects of Polarizability on the Hydration of the Chloride Ion. Journal of Physical Chemistry, 1996. 100(29): p. 11934-11943. 
174. M. Sprik, M.L. Klein, and K. Watanabe, Solvent Polarization and Hydration of the Chlorine Anion. Journal of Physical Chemistry, 1990. 94(16): p. 6483-6488.

175. L.X. Dang, J.E. Rice, J. Caldwell, and P.A. Kollman, Ion Solvation in Polarizable Water Molecular-Dynamics Simulations. Journal of the American Chemical Society, 1991. 113(7): p. 2481-2486.

176. B. Roux, B. Prodhom, and M. Karplus, Ion-Transport in the Gramicidin Channel Molecular-Dynamics Study of Single and Double Occupancy. Biophysical Journal, 1995. 68(3): p. 876-892.

177. I.S. Joung and T.E. Cheatham, Determination of Alkali and Halide Monovalent Ion Parameters for Use in Explicitly Solvated Biomolecular Simulations. Journal of Physical Chemistry B, 2008. 112(30): p. 9020-9041.

178. G.L. Warren and S. Patel, Electrostatic Properties of Aqueous Salt Solution Interfaces: A Comparison of Polarizable and Nonpolarizable Ion Models. Journal of Physical Chemistry B, 2008. 112(37): p. 11679-93.

179. M. Masia, M. Probst, and R. Rey, On the Performance of Molecular Polarization Methods. I. Water and Carbon Tetrachloride Close to a Point Charge. Journal of Chemical Physics, 2004. 121(15): p. 7362-7378.

180. M. Masia, M. Probst, and R. Rey, On the Performance of Molecular Polarization Methods. II. Water and Carbon Tetrachloride Close to a Cation. Journal of Chemical Physics, 2005. 123(16): p. 164505-164518.

181. X. Li and Z.Z. Yang, Hydration of Li-Ion in Atom-Bond Electronegativity Equalization Method-7P Water: A Molecular Dynamics Simulation Study. Journal of Chemical Physics, 2005. 122(8): p84514. 
182. Z.Z. Yang and X. Li, Molecular-Dynamics Simulations of Alkaline-Earth Metal Cations in Water by Atom-Bond Electronegativity Equalization Method Fused into Molecular Mechanics. Journal of Chemical Physics, 2005. 123(9): p094507.

183. P. Jungwirth and D.J. Tobias, Chloride Anion on Aqueous Clusters, at the Air-Water Interface, and in Liquid Water: Solvent Effects on Cl Polarizability. Journal of Physical Chemistry A, 2002. 106(2): p. 379-383.

184. G. Archontis, E. Leontidis, and G. Andreou, Attraction of lodide lons by the Free Water Surface, Revealed by Simulations with a Polarizable Force Field Based on Drude Oscillators. Journal of Physical Chemistry B, 2005. 109(38): p. 17957-17966.

185. G. Archontis and E. Leontidis, Dissecting the Stabilization of lodide at the Air-Water Interface into Components: A Free Energy Analysis. Chemical Physics Letters, 2006. 420(1-3): p. 199-203.

186. M.A. Brown, R. D'Auria, I.F.W. Kuo, M.J. Krisch, D.E. Starr, H. Bluhm, D.J. Tobias, and J.C. Hemminger, Ion Spatial Distributions at the Liquid-Vapor Interface of Aqueous Potassium Fluoride Solutions. Physical Chemistry Chemical Physics, 2008. 10(32): p. 4778-4784.

187. X.W. Wang, H. Watanabe, M. Fuji, and M. Takahashi, Molecular Dynamics Simulation of $\mathrm{NaCl}$ at the Air/Water Interface with Shell Model. Chemical Physics Letters, 2008. 458(13): p. 235-238.

188. A. Grossfield, Dependence of Ion Hydration on the Sign of the lon's Charge. Journal of Chemical Physics, 2005. 122(2): p. 024506.

189. P.S. Bagus, K. Hermann, and J.C.W. Bauschlicher, A New Analysis of Charge Transfer and Polarization for Ligand--Metal Bonding: Model Studies of $\mathrm{Al}_{4} \mathrm{Co}$ and $\mathrm{Al}_{4} \mathrm{NH}_{3}$. The Journal of Chemical Physics, 1984. 80(9): p. 4378-4386. 
190. J.-P. Piquemal, L. Perera, G.A. Cisneros, P. Ren, L.G. Pedersen, and T.A. Darden, Towards Accurate Solvation Dynamics of Divalent Cations in Water Using the Polarizable Amoeba Force Field: From Energetics to Structure. The Journal of Chemical Physics, 2006. 125(5): p. 054511-7.

191. M. Devereux, M.C. van Severen, O. Parisel, J.P. Piquemal, and N. Gresh, Role of Cation Polarization in Holo- and Hemi-Directed $[\mathrm{Pb}(\mathrm{H} 2 \mathrm{O})(\mathrm{N})]^{2+}$ Complexes and Development of a $\mathrm{Pb}^{2+}$ Polarizable Force Field. Journal of Chemical Theory and Computation, 2011. 7(1): $\mathrm{p}$. 138-147.

192. T.P. Straatsma and J.A. McCammon, Free Energy Evaluation from Molecular Dynamics Simulations Using Force Fields Including Electronics Polarization. Chemical Physics Letters, 1991. 177(4-5): p. 433-440.

193. D.L. Mobley, C.I. Bayly, M.D. Cooper, M.R. Shirts, and K.A. Dill, Small Molecules Hydration Free Energies in Explicit Solvent: An Extensive Test of Fixed-Charge Atomistic Simulations. Journal of Chemical Theory and Computation, 2009. 5: p. 350-359.

194. D.L. Mobley, E. Dumont, J.D. Chodera, and K.A. Dill, Comparison of Charge Models for Fixed-Charge Force Fields: Small-Molecule Hydration Free Energies in Explicit Solvent. Journal of Physical Chemistry B, 2007. 111(9): p. 2242-2254.

195. R.C. Rizzo, T. Aynechi, D.A. Case, and I.D. Kuntz, Estimation of Absolute Free Energies of Hydration Using Continuum Methods: Accuracy of Partial, Charge Models and Optimization of Nonpolar Contributions. Journal of Chemical Theory and Computation, 2006. 2(1): p. 128-139.

196. M.R. Shirts and V.S. Pande, Solvation Free Energies of Amino Acid Side Chain Analogs for Common Molecular Mechanics Water Models. Journal of Chemical Physics, 2005. 122(13): p. 134508. 
197. X.Q. Sun and L.X. Dang, Computational Studies of Aqueous Interfaces of $R b B r$ Salt Solutions. Journal of Chemical Physics, 2009. 130(21): p. 124709-124713.

198. V.M. Anisimov, I.V. Vorobyov, B. Roux, and A.D. MacKerell, Polarizable Empirical Force Field for the Primary and Secondary Alcohol Series Based on the Classical Drude Model. Journal of Chemical Theory and Computation, 2007. 3(6): p. 1927-1946.

199. A. Hesselmann and G. Jansen, First-Order Intermolecular Interaction Energies from KohnSham Orbitals. Chemical Physics Letters, 2002. 357(5-6): p. 464-470.

200. J.C. Wu, G. Chattre, and P. Ren, Automation of AMOEBA Polarizable Force Field Parameterization for Small Molecules. Theoretical Chemistry Accounts, 2012. 131(3): p. 1138-1148.

201. T. Ogawa, N. Kurita, H. Sekino, O. Kitao, and S. Tanaka, Consistent Charge Equilibration (CQEQ) Method: Application to Amino Acids and Crambin Protein. Chemical Physics Letters, 2004. 397(4-6): p. 382-387.

202. E. Harder, B.C. Kim, R.A. Friesner, and B.J. Berne, Efficient Simulation Method for Polarizable Protein Force Fields: Application to the Simulation of BPTI in Liquid. Journal of Chemical Theory and Computation, 2005. 1(1): p. 169-180.

203. P. Llinas, M. Masella, T. Stigbrand, A. Menez, E.A. Stura, and M.H. Le Du, Structural Studies of Human Alkaline Phosphatase in Complex with Strontium: Implication for Its Secondary Effect in Bones. Protein Science, 2006. 15(7): p. 1691-1700.

204. Z.X. Wang, W. Zhang, C. Wu, H.X. Lei, P. Cieplak, and Y. Duan, Erratum - Strike a Balance: Optimization of Backbone Torsion Parameters of AMBER Polarizable Force Field for Simulations of Proteins and Peptides. Journal of Computational Chemistry, 2006. 27(6): p. 781-790. 
205. Z.Z. Yang and Q. Zhang, Study of Peptide Conformation in Terms of the ABEEM/MM Method. Journal of Computational Chemistry, 2006. 27(1): p. 1-10.

206. Q.M. Guan, B.Q. Cui, D.X. Zhao, L.D. Gong, and Z.Z. Yang, Molecular Dynamics Study on BPTI Aqueous Solution by ABEEM/MM Fluctuating Charge Model. Chinese Science Bulletin, 2008. 53(8): p. 1171-1174.

207. B.Q. Cui, Q.M. Guan, L.D. Gong, D.X. Zhao, and Z.Z. Yang, Studies on the Heme Prosthetic Group's Geometry by ABEEM/MM Method. Chemical Journal of Chinese UniversitiesChinese, 2008. 29(3): p. 585-590.

208. B. de Courcy, J.P. Piquemal, C. Garbay, and N. Gresh, Polarizable Water Molecules in Ligand-Macromolecule Recognition. Impact on the Relative Affinities of Competing Pyrrolopyrimidine Inhibitors for FAK Kinase. Journal of the American Chemical Society, 2010. 132(10): p. 3312-3320.

209. B. de Courcy, L.G. Pedersen, O. Parisel, N. Gresh, B. Silvi, J. Pilme, and J.P. Piquemal, Understanding Selectivity of Hard and Soft Metal Cations within Biological Systems Using the Subvalence Concept. I. Application to Blood Coagulation: Direct Cation-Protein Electronic Effects Vs. Indirect Interactions through Water Networks. Journal of Chemical Theory and Computation, 2010. 6(4): p. 1048-1063.

210. B. de Courcy, J.P. Dognon, C. Clavaguera, N. Gresh, and J.P. Piquemal, Interactions within the Alcohol Dehydrogenase Zn(II)-Metalloenzyme Active Site: Interplay between Subvalence, Electron Correlation/Dispersion, and Charge Transfer/Induction Effects. International Journal of Quantum Chemistry, 2011. 111(6): p. 1213-1221.

211. A. De La Lande, D.R. Salahub, J. Maddaluno, A. Scemama, J. Pilme, O. Parisel, H. Gerard, M. Caffarel, and J.P. Piquemal, Rapid Communication Spin-Driven Activation of Dioxygen 
in Various Metalloenzymes and Their Inspired Models. Journal of Computational Chemistry, 2011. 32(6): p. 1178-1182.

1381

212. A.G. Donchev, N.G. Galkin, A.A. Illarionov, O.V. Khoruzhii, M.A. Olevanov, V.D. Ozrin, L.B. Pereyaslavets, and V.I. Tarasov, Assessment of Performance of the General Purpose Polarizable Force Field QMPFF3 in Condensed Phase. Journal of Computational Chemistry, 2008. 29(8): p. 1242-1251.

213. E. Harder, A.D. MacKerell, and B. Roux, Many-Body Polarization Effects and the Membrane Dipole Potential. Journal of the American Chemical Society, 2009. 131(8): p. $2760-2761$.

214. J.E. Davis, O. Raharnan, and S. Patel, Molecular Dynamics Simulations of a DMPC Bilayer Using Nonadditive Interaction Models. Biophysical Journal, 2009. 96(2): p. 385-402.

215. B. Roux and T. Simonson, Implicit Solvent Models. Biophysical Chemistry, 1999. 78(1-2): p. 1-20.

216. N.A. Baker, Improving Implicit Solvent Simulations: A Poisson-Centric View. Current Opinion in Structural Biology, 2005. 15(2): p. 137-143.

217. N.A. Baker, Reviews in Computational Chemistry, K.B. Lipkowitz, R. Lorter, and T. Cundari. Vol. 21. 2005, New York: Wiley-VCH Inc., Biomolecular Applications of PoissonBoltzmann Methods.

218. N.A. Baker, Poisson-Boltzmann Methods for Biomolecular Electrostatics. Methods in Enzymology, 2004. 383: p. 94-118.

219. J. Tomasi, B. Mennucci, and R. Cammi, Quantum Mechanical Continuum Solvation Models. Chemical Reviews, 2005. 105(8): p. 2999-3093.

220. J. Tomasi, Thirty Years of Continuum Solvation Chemistry: A Review, and Prospects for the near Future. Theoretical Chemistry Accounts, 2004. 112(4): p. 184-203. 
1403

1404

1405

1406

1407

1408

1409

1410

1411

1412

1413

1414

1415

1416

1417

1418

1419

1420

1421

1422

1424

1425

1426

221. A. Klamt and G. Schuurmann, COSMO - a New Approach to Dielectric Screening in Solvents with Explicit Expressions for the Screening Energy and Its Gradient. Journal of the Chemical Society: Perkin Transactions 2, 1993(5): p. 799-805.

222. C.J. Cramer and D.G. Truhlar, Implicit Solvation Models: Equilibria, Structure, Spectra, and Dynamics. Chemical Reviews, 1999. 99(8): p. 2161-2200.

223. J.R. Maple, Y.X. Cao, W.G. Damm, T.A. Halgren, G.A. Kaminski, L.Y. Zhang, and R.A. Friesner, A Polarizable Force Field and Continuum Solvation Methodology for Modeling of Protein-Ligand Interactions. Journal of Chemical Theory and Computation, 2005. 1(4): p. 694-715.

224. J.-F. Truchon, A. Nicholls, R.I. Iftimie, B. Roux, and C.I. Bayly, Accurate Molecular Polarizabilities Based on Continuum Electrostatics. Journal of Chemical Theory and Computation, 2008. 4(9): p. 1480-1493.

225. J.-F. Truchon, A. Nicholls, B. Roux, R.I. Iftimie, and C.I. Bayly, Integrated Continuum Dielectric Approaches to Treat Molecular Polarizability and the Condensed Phase: Refractive Index and Implicit Solvation. Journal of Chemical Theory and Computation, 2009. 5(7): p. 1785-1802.

226. T. Yang, J.C. Wu, C. Yan, Y. Wang, R. Luo, M.B. Gonzales, K.N. Dalby, and P. Ren, Virtual Screening Using Molecular Simulations. Proteins: Structure, Function, and Bioinformatics, 2011. 79(6): p. 1940-1951.

227. D.-X. Zhao, L. Yu, L.-D. Gong, C. Liu, and Z.-Z. Yang, Calculating Solvation Energies by Means of a Fluctuating Charge Model Combined with Continuum Solvent Model. The Journal of Chemical Physics, 2011. 134(19): p. 194115.

228. A. McCoy, Liking Likelihood. Acta Crystallographica Section D, 2004. 60(12 Part 1): p. 2169-2183. 
229. W.I. Weis, A.T. Brunger, J.J. Skehel, and D.C. Wiley, Refinement of the Influenza-Virus Hemagglutinin by Simulated Annealing. Journal of Molecular Biology, 1990. 212(4): p. 737-761.

230. L. Moulinier, D.A. Case, and T. Simonson, Reintroducing Electrostatics into Protein X-Ray Structure Refinement: Bulk Solvent Treated as a Dielectric Continuum. Acta Crystallographica Section D-Biological Crystallography, 2003. 59: p. 2094-2103.

231. C. Sagui and T.A. Darden, Molecular Dynamics Simulations of Biomolecules: Long-Range Electrostatic Effects. Annual Review of Biophysics and Biomolecular Structure, 1999. 28: p. 155-179.

232. P.P. Ewald, Die Berechnung Optischer Und Elektrostatischer Gitterpotentiale. Annalen der Physik, 1921. 369(3): p. 253-287.

233. W. Smith, Point Multipoles in the Ewald Summation (Revisited). CCP5 Information Quaterly, 1982.4(13).

234. P. Ren and J.W. Ponder, Polarizable Atomic Multipole Water Model for Molecular Mechanics Simulation. Journal of Physical Chemistry B, 2003. 107(24): p. 5933-5947.

235. T. Darden, D. York, and L. Pedersen, Particle-Mesh Ewald - an N Log(N) Method for Ewald Sums in Large Systems. Journal of Chemical Physics, 1993. 98(12): p. 10089-10092.

236. U. Essmann, L. Perera, M.L. Berkowitz, T. Darden, H. Lee, and L.G. Pedersen, A Smooth Particle-Mesh Ewald Method. Journal of Chemical Physics, 1995. 103(19): p. 8577-8593.

237. C. Sagui, L.G. Pedersen, and T.A. Darden, Towards an Accurate Representation of Electrostatics in Classical Force Fields: Efficient Implementation of Multipolar Interactions in Biomolecular Simulations. Journal of Chemical Physics, 2004. 120(1): p. 73-87. 
238. T.D. Fenn, M.J. Schnieders, A.T. Brunger, and V.S. Pande, Polarizable Atomic Multipole XRay Refinement: Hydration Geometry and Application to Macromolecules. Biophysical Journal, 2010. 98(12): p. 2984-2992.

239. T.D. Fenn, M.J. Schnieders, M. Mustyakimov, C. Wu, P. Langan, V.S. Pande, and A.T. Brunger, Reintroducing Electrostatics into Macromolecular Crystallographic Refinement: Application to Neutron Crystallography and DNA Hydration. Structure, 2011. 19(4): p. 523-533.

240. M.J. Schnieders, T.S. Kaoud, C. Yan, K.N. Dalby, and P. Ren, Computational Insights for the Discovery of Non-ATP Competitive Inhibitors of MAP Kinases. Current Pharmaceutical Design, 2012. 18(9): p. 1173-1185.

241. V.B. Chen, W.B. Arendall, J.J. Headd, D.A. Keedy, R.M. Immormino, G.J. Kapral, L.W. Murray, J.S. Richardson, and D.C. Richardson, MolProbity: All-Atom Structure Validation for Macromolecular Crystallography. Acta Crystallographica Section D, 2009. 66: p. 1221.

242. J. Maddox, Crystals from First Principles. Nature, 1998. 335(6187): p. 201.

243. G.M. Day, T.G. Cooper, A.J. Cruz-Cabeza, K.E. Hejczyk, H.L. Ammon, S.X.M. Boerrigter, J.S. Tan, R.G. Della Valle, E. Venuti, J. Jose, S.R. Gadre, G.R. Desiraju, T.S. Thakur, B.P. van Eijck, J.C. Facelli, V.E. Bazterra, M.B. Ferraro, D.W.M. Hofmann, M.A. Neumann, F.J.J. Leusen, J. Kendrick, S.L. Price, A.J. Misquitta, P.G. Karamertzanis, G.W.A. Welch, H.A. Scheraga, Y.A. Arnautova, M.U. Schmidt, J. van de Streek, A.K. Wolf, and B. Schweizer, Significant Progress in Predicting the Crystal Structures of Small Organic Molecules - A Report on the Fourth Blind Test. Acta Crystallographica Section B, 2009. 65: p. 107-125.

244. D.A. Bardwell, C.S. Adjiman, Y.A. Arnautova, E. Bartashevich, S.X.M. Boerrigter, D.E. Braun, A.J. Cruz-Cabeza, G.M. Day, R.G. Della Valle, G.R. Desiraju, B.P. van Eijck, J.C. 
Facelli, M.B. Ferraro, D. Grillo, M. Habgood, D.W.M. Hofmann, F. Hofmann, K.V.J. Jose, P.G. Karamertzanis, A.V. Kazantsev, J. Kendrick, L.N. Kuleshova, F.J.J. Leusen, A.V. Maleev, A.J. Misquitta, S. Mohamed, R.J. Needs, M.A. Neumann, D. Nikylov, A.M. Orendt, R. Pal, C.C. Pantelides, C.J. Pickard, L.S. Price, S.L. Price, H.A. Scheraga, J. van de Streek, T.S. Thakur, S. Tiwari, E. Venuti, and I.K. Zhitkov, Towards Crystal Structure Prediction of Complex Organic Compounds - A Report on the Fifth Blind Test. Acta Crystallographica Section B, 2011. 67(6): p. 535-551.

245. W. Cabri, P. Ghetti, G. Pozzi, and M. Alpegiani, Polymorphisms and Patent, Market, and Legal Battles: Cefdinir Case Study. Organic Process Research \& Development, 2006. 11(1): p. 64-72.

246. S.L. Price and L.S. Price, Computational Polymorph Prediction, in Solid State Characterization of Pharmaceuticals. 2011, John Wiley \& Sons, Ltd. p. 427-450.

247. S.L. Price, From Crystal Structure Prediction to Polymorph Prediction: Interpreting the Crystal Energy Landscape. Physical Chemistry Chemical Physics, 2008. 10(15): p. 19962009.

248. P. Ren, M. Marucho, J. Zhang, and N.A. Baker, Biomolecular Electrostatics and Solvation: A Computational Perspective. Quarterly Reviews of Biophysics, 2011. 45(4): p. 427-491.

249. G.W.A. Welch, P.G. Karamertzanis, A.J. Misquitta, A.J. Stone, and S.L. Price, Is the Induction Energy Important for Modeling Organic Crystals? Journal of Chemical Theory and Computation, 2008. 4(3): p. 522-532.

250. B. Civalleri, C.M. Zicovich-Wilson, L. Valenzano, and P. Ugliengo, B3LYP Augmented with an Empirical Dispersion Term (B3LYP-D*) as Applied to Molecular Crystals. CrystEngComm, 2008. 10(4): p. 405-410. 
251. S.L. Price, Quantifying Intermolecular Interactions and Their Use in Computational Crystal Structure Prediction. CrystEngComm, 2004. 6(61): p. 344-353.

252. A. Gavezzotti, Calculation of Intermolecular Interaction Energies by Direct Numerical Integration over Electron Densities. I. Electrostatic and Polarization Energies in Molecular Crystals. Journal of Physical Chemistry B, 2002. 106(16): p. 4145-4154.

253. A. Gavezzotti, Calculation of Intermolecular Interaction Energies by Direct Numerical Integration over Electron Densities. 2. An Improved Polarization Model and the Evaluation of Dispersion and Repulsion Energies. Journal of Physical Chemistry B, 2003. 107(10): p. 2344-2353.

254. W.T.M. Mooij, B.P. van Eijck, and J. Kroon, Transferable Ab Initio Intermolecular Potentials. 2. Validation and Application to Crystal Structure Prediction. Journal of Physical Chemistry A, 1999. 103(48): p. 9883-9890.

255. P. Ren and J.W. Ponder, Consistent Treatment of Inter- and Intramolecular Polarization in Molecular Mechanics Calculations. Journal of Computational Chemistry, 2002. 23(16): p. 1497-1506.

256. A.J. Misquitta and A.J. Stone, Accurate Induction Energies for Small Organic Molecules: 1. Theory. Journal of Chemical Theory and Computation, 2007. 4(1): p. 7-18.

257. A.J. Misquitta, A.J. Stone, and S.L. Price, Accurate Induction Energies for Small Organic Molecules. 2. Development and Testing of Distributed Polarizability Models against SAPT(DFT) Energies. Journal of Chemical Theory and Computation, 2007. 4(1): p. 19-32.

258. C. Ouvrard and S.L. Price, Toward Crystal Structure Prediction for Conformationally Flexible Molecules: The Headaches Illustrated by Aspirin. Crystal Growth \& Design, 2004. 4(6): p. 1119-1127. 
1521 